\title{
Efficient and Privacy-Preserving Cryptographic Key Derivation from Continuous Sources
}

\author{
Enrique Argones Rúa, Aysajan Abidin, Roel Peeters, Jac Romme
}

\begin{abstract}
The procedure for extracting a cryptographic key from noisy sources, such as biometrics and Physically Uncloneable Functions (PUFs), is known as Fuzzy Extractor (FE). Although FE constructions deal with discrete sources, most noisy sources are continuous. In the continuous case, it is required to transform the source to a discrete one. We introduce a (i) model-based uncoupling construction that deals directly with the continuous noisy source and produces helper data uncoupling the discrete representation from the noisy source, guaranteeing the diversity of the discrete representation, and making it more robust; and a (ii) strengthened uncoupled fuzzy extractor, suitable for privacy-preserving applications, that integrates an additional fixed authentication factor and obtains a key uncoupled to the noisy sources and unlinkable helper data. We present optimal model-based uncoupling constructions for Gaussian sources. Specifically, we show how to extract (i) one or multiple bits from single Gaussian source, (ii) one bit from several unreliable Gaussian sources; and provide a general procedure to obtain an optimal uncoupled FE from Gaussian source(s). Our experiments show that the proposed constructions achieve much higher security levels for wide operational scenarios, approximately doubling the obtained effective key length without affecting false rejection rates.
\end{abstract}

\section{INTRODUCTION}

W ITH the Internet of Things comes an ever growing number of devices that need to communicate securely with each other. To set up secure communication among all these devices, solutions increasingly make use of noisy sources such as biometrics (including behaviometrics) to authenticate users; Physically Uncloneable Functions (PUFs) for deriving inherent device-unique keys without the need for programming these devices; and sensor inputs to derive short lived session keys, e.g., to exchange data with devices that are in the same physical environment.

The construction for extracting cryptographic keys from noisy sources is known as Fuzzy Extractor (FE) [1]. In general, an FE consists of generation and reproduction procedures. In the generation procedure, one derives a stable string from an inherently noisy discrete source, which is indistinguishable from a random string, and helper data. In the reproduction procedure, one recovers the same string from the same source and the helper data computed during the generation procedure.

Enrique Argones Rúa, Aysajan Abidin, and Roel Peeters, are with the imec-Computer Security and Industrial Cryptography, Departement Elektrotechniek - ESAT, KULeuven. Jac Romme is with imec, The Netherlands.

This work was supported by the Flemish government through the FWO SBO project SPITE S002417N and by imec through the Security \& Privacy Centre projects on Biometrics \& Authentication and Secure Distance Bounding.

Manuscript received March, 22, 2019
However, FEs do not deal with continuous sources, which are the most common in the real world.

In this paper, we first propose a model-based uncoupling construction, which extracts from a continuous source a discrete representation and metadata useful for reproduction. This construction is a generalisation of the Helper Data Schemes proposed by Groot et al. [2]. The metadata is essential for ensuring the maximum reliability of the discrete representation, which is uncoupled form the continuous source. We propose a complete procedure for ensuring minimum number of errors in the reproduction procedure when sampling from the genuine source(s), but full entropy when sampling from the general population in the case of Gaussian sources.

Note that the metadata extracted within the model-based uncoupling construction discloses identifiable information about the Gaussian source: it is only guaranteed that a bounded amount of entropy remains hidden, disclosing the rest about the continuous source. For the derivation of one-time session keys, e.g., based on sensor data, which can be assumed to be public and sufficiently different from one realisation to another (due to highly fluctuating sources), the information leakage on the continuous source is not a concern. The metadata could thus remain unprotected. However, the information leakage becomes a privacy concern when the signal itself may be considered as sensitive information, e.g., biometrics, or in general when the FE is used to derive cryptographic keys based on non-variant noisy inputs. In these cases, the metadata enables cross-linking devices (PUFs) or even users (biometrics) [3] among different applications. For these cases, we propose to use a second factor in the strengthened uncoupled fuzzy extractor to ensure complete unlinkability among the stored information in different instances of templates from the same noisy sources. This construction is consistent with the existing standard recommendations for high security access control systems [4]. The uncoupling between the discrete representation and the continuous noisy source ensures the maximum diversity of the obtained discrete representations, enabling revocation and renewal of the obtained templates. These properties make the strengthened uncoupled fuzzy extractor suitable for privacy-preserving applications.

Concretely, our contributions are as follows:

- The formalization of the Strengthened Uncoupled Fuzzy Extractor, which (i) obtains a discrete representation of the biometric model uncoupled from the biometric model by means of the Model-based Uncoupling Construction, then (ii) protects the produced metadata, linkable with the continuous source, using a fixed authentication factor to avoid the disclosure of any information about the 
protected template, and (iii) derives helper data which allows verification and does not disclose information about the continuous source.

- Introduction and theoretical analysis of optimal Modelbased Uncoupling Constructions for Gaussian sources, using zero-leakage metadata extraction procedures for reliable Gaussian sources and optimal feature combination for unreliable ones, allowing maximum reliability of the discrete representation for genuine attempts while ensuring uniform distribution for impostor attempts.

- Description of the complete design process of a strengthened uncoupled fuzzy extractor for Gaussian features, supported by the previous model-based uncoupling constructions, allowing to close the gap from Gaussian features to fuzzy extractors.

Finally, we conducted an empirical evaluation in different use cases of the proposed constructions for key agreement based on shared noisy radio frequency (RF) channel representations. This evaluation shows the advantages of using the proposed constructions over using uniform quantization, which is nowadays the most extended approach, in terms of effective key length and False Rejection Rate (FRR).

\section{RELATED WORK}

Since the publication of Juels and Wattenberg's fuzzy commitment scheme [5], there have been many advances for obtaining cryptographic keys from noisy sources. Dodis et al. [1] formalized the well-known FE scheme. However, a degradation in performance is commonly observed when integrating the fuzzy extraction in verification chains. The extraction of robust biometric features suitable for the construction of FEs has been the focus of some works in biometrics, such as the work by Tong et al. [6]. Another example can be found in [7], where eigenmodel features are used within a fuzzy commitment scheme for online signature verification, but still the degradation in performance is observable even after the fusion of features before the quantization. Billeb et al. [8] proposed to use the same type of features for speaker verification, and then apply feature selection based on reliability measure for the inclusion of the obtained binary features in the FE. Van der Veen et al. [9] proposed the use of a fuzzy commitment scheme for face verification, also highlighting the importance of selecting the most reliable bits before including them in the FE. This trend can be observed in other works on biometric template protection for other modalities such as iris [10]. The use of sophisticated error correcting codes such as Turbo-Codes was explored by Maiorana et al. [11] for the features extracted in [7].

FEs have also become increasingly important in PUF-based key generation. One challenging task in IoT and/or embedded devices is the generation and storage of cryptographic keys. Such devices have little protection against unauthorized access to their non-volatile memory. PUFs together with an FE can offer a solution to the above challenge. The idea is to use an FE to first derive a cryptographic key and helper data from the PUF response (which is typically a bitstring), and then using the helper data together with a noisy PUF response to recover the key again. Note that depending on the number of challenges that the PUF can process, it is categorized as either strong PUF or weak PUF. While the former can process large number (ideally, exponential in the length of the response) of challenges, the latter can only support a small number of challenges (usually, only one challenge). As such, strong PUFs are mostly used for authentication, while weak PUFs are for key derivation. There has been a lot of research on PUF-based key generation using FEs [12], [13], [14], [15], [16], [17]. However, these PUF-based key derivation solutions only use FEs to derive a key from the (possibly) noisy PUF response, and do not take into account the physical measurements that produce the PUF response. Therefore, our parametrized binarisation approach can be applied to quantize the physical measurements inside weak PUFs to reduce bit error rates.

Ignatenko [18] analyzed the information loss due to the helper data in FEs, although her work mainly focused on dealing with quantized biometrics. The combination with other authentication factors was analyzed from an information theoretic point of view in [19], again dealing with fixed quantization schemes for the biometrics. Verbitskiy et al. [20] analyzed the problem of fuzzy extraction from continuous sources, pointing out that the distribution of the continuous features can be used to derive uniformly distributed keys. Groot et al. [2] showed a zero-leakage helper data scheme for fuzzy extraction for general distributions of continuous features, which is of practical interest for the construction of FEs when dealing with reliable continuous features, though they do not cover the case of dealing with unreliable features, where a combination of these is needed.

\section{StREngthened UnCOUPLEd FuzZy ExtRactor}

This section formally introduces the strengthened uncoupled fuzzy extractor and presents a practical implementation based on symmetric encryption, universal one-way hash functions and error correcting codes, as defined in Appendix A. Below we recall necessary concepts and naming conventions:

A population $O=\left\{O_{1}, \ldots, O_{P}\right\}$ is a set of a variable number of individuals (persons or objects) that share some common measurable characteristics.

Given a population $O$ of individuals, a random characterization $Q$ of an individual $O \in O$ is a random variable obtained as a noisy measurement of some characteristics of the individual which are common among the individuals in the population: $Q=$ measure $(O) \in Q$, where $Q$ is the measurements' range. We denote by $q \leftarrow Q$ a realization of the random characterizarion, or sample. Let $\mathcal{M}$ be a model space, in which a model $M \in \mathcal{M}$ of an individual $O \in O$ is a set of random parameters describing a random characterization of $O$. We denote by $m \leftarrow M$ a realization of the model $M$, or template.

\section{A. Formal Constructions}

Now we present the formal constructions, first those dealing with the models of the noisy sources (the individuals within a population), and then the strengthened uncoupled fuzzy 
extractor, integrating the fuzzy extraction, the fuzzy model characterisations, and the fixed authentication factor.

Definition III.1 (Model estimation function). A model estimation function model : $Q^{E} \rightarrow \mathcal{M}$ maps a vector of $E$ enrolment samples $\left[q^{1}, \ldots, q^{E}\right]$ from a random characterization $Q$ of an individual $O$ to a template of the individual $m=\operatorname{model}\left(q^{1}, \ldots, q^{E}\right)$.

Definition III.2 (Model-based verifier). A function $\operatorname{ver}_{\mathcal{M}}$ : $\mathcal{M} \times Q^{V} \rightarrow\{0,1\}$ mapping a template $m_{i}$ of an individual $O_{i}$ and a vector $\boldsymbol{q}_{j}=\left[q_{j}^{1}, \ldots, q_{j}^{V}\right]$ of $V$ verification samples from $Q_{j}=$ measure $\left(O_{j}\right)$ to a decision on whether $O_{i}$ and $O_{j}$ are the same is called a $(Q, \mathcal{M}, V$, FAR, FRR)-model-based verifier, characterized by the following error probabilities:

$$
\begin{aligned}
& \mathrm{FRR}=\operatorname{Pr}\left\{\operatorname{ver}_{\mathcal{M}, Q^{V}}\left(m_{i}, \boldsymbol{q}_{j}\right)=0 \mid O_{i}=O_{j}\right\}, \\
& \mathrm{FAR}=\operatorname{Pr}\left\{\operatorname{ver}_{\mathcal{M}, Q^{V}}\left(m_{i}, \boldsymbol{q}_{j}\right)=1 \mid O_{i} \neq O_{j}\right\} .
\end{aligned}
$$

Definition III.3 (Model-based uncoupling construction). Let $\{\mathrm{ME}, \mathrm{PM}\}$ be a construction comprising:

(i) a metadata extractor ME : $\mathcal{M} \times C^{n} \rightarrow \mathcal{P}$, and

(ii) a parametrized mapping $\mathrm{PM}: Q^{V} \times \mathcal{P} \rightarrow C^{n}$,

where $\mathcal{P}$ is the metadata domain. If, for a given model $M$ of an individual $O$, a random string $C \in C^{n}$ independent from $M$, where $C$ is a finite alphabet, and the metadata $P_{M, C}=$ $\operatorname{ME}(M, C)$, the following equations hold:

$$
\mathrm{I}\left(\boldsymbol{C}, P_{M, \boldsymbol{C}}\right)=0, \quad \mathrm{H}\left(M \mid P_{M, \boldsymbol{C}}\right) \geq n,
$$

where $\mathrm{I}(\cdot, \cdot)$ is the mutual information and $\mathrm{H}(\cdot \cdot \cdot)$ is the conditional entropy, then $\{\mathrm{ME}, \mathrm{PM}\}$ is a $(Q, \mathcal{M}, \mathcal{P}, C, V, n)$-modelbased uncoupling construction.

Definition III.4 (Uncoupled model-based verifier). A function ver $_{\mathrm{ME}, \mathrm{PM}}: \mathcal{M} \times Q^{V} \rightarrow\{0,1\}$ defined as:

$$
\operatorname{ver}_{\mathrm{ME}, \mathrm{PM}}\left(m_{i}, \boldsymbol{q}_{j}\right)= \begin{cases}1 & \text { if d }(\boldsymbol{c}, \tilde{\boldsymbol{c}}) \leq t, \\ 0 & \text { otherwise }\end{cases}
$$

where $m_{i} \in \mathcal{M}$ is a template of an individual $O_{i}, \boldsymbol{q}_{j}=$ $\left[q_{j}^{1}, \ldots, q_{j}^{V}\right]$ is a vector of $V$ verification samples from $Q_{j}=$ measure $\left(O_{j}\right), c \in C^{n}$ is a random string independent from $m_{i}, \mathrm{~d}(\cdot \cdot \cdot)$ is a distance in $C^{n},\{\mathrm{ME}, \mathrm{PM}\}$ is an $(Q, \mathcal{M}, \mathcal{P}, C, V, n)$-model-based uncoupling construction, $p_{m_{i}, \boldsymbol{c}}=\operatorname{ME}\left(m_{i}, \boldsymbol{c}\right)$, and $\tilde{\boldsymbol{c}}=\operatorname{PM}\left(\boldsymbol{q}_{j}, p_{m_{i}, \boldsymbol{c}}\right)$, is called a $(Q, \mathcal{M}, \mathcal{P}, C, V, n, t$, FAR, FRR)-uncoupled model-based verifier characterized by the following error probabilities:

$$
\begin{aligned}
& \text { FRR }=\operatorname{Pr}\left\{\operatorname{ver}_{\mathrm{ME}, \mathrm{PM}}\left(m_{i}, \boldsymbol{q}_{j}\right)=0 \mid O_{i}=O_{j}\right\}, \\
& \mathrm{FAR}=\operatorname{Pr}\left\{\operatorname{ver}_{\mathrm{ME}, \mathrm{PM}}\left(m_{i}, \boldsymbol{q}_{j}\right)=1 \mid O_{i} \neq O_{j}\right\} .
\end{aligned}
$$

Definition III.5 (Strengthened uncoupled fuzzy extractor). The procedures (Gen, Rep) constitute a $(\mathcal{F S}, Q, \mathcal{M}, \mathcal{P} \mathcal{S}, C, V, n, k$, FAR, FRR)-uncoupled extractor when the following conditions hold:

1) Gen is a probabilistic generation procedure Gen : $\mathcal{F S} \times$ $\mathcal{M} \rightarrow \mathcal{P S} \times C^{k}$ which on input $\left(f s, m_{j}\right)$, where $m_{i}$ is a template from an individual $O_{i}$, and $f_{s} \in \mathcal{F} \mathcal{S}$ is a fixed secret sampled from the random variable $F S$ independent from $M_{i}$, outputs a public string $p s \leftarrow P S \in \mathcal{P S}$, and a secret uniformly random string $s \boldsymbol{s} \leftarrow S S \sim \mathrm{U}_{C^{k}}$, hereafter $U_{\star}$ is the uniform distribution over the domain $\star$, such that:

(i) $\mathrm{I}\left(P S, M_{i}\right)=0$.

(ii) $\mathrm{I}\left(S S, M_{i}\right)=0$.

(iii) $\mathrm{I}\left(M_{i},(F S, P S)\right) \leq \mathrm{H}\left(M_{i}\right)-n$.

2) Rep is a function defined as $\operatorname{Rep}: \mathcal{F S} \times \mathcal{P} \mathcal{S} \times Q^{V} \rightarrow$ $C^{k} \cup\{\emptyset\}$ which takes as input $\left(f s, p s, \boldsymbol{q}_{j}\right)$, and given that $(p s, \boldsymbol{s} \boldsymbol{s})=\operatorname{Gen}\left(f s, m_{i}\right)$, where $m_{i} \in \mathcal{M}$ is a template of an individual $O_{j}$, the following equations hold:

(i) $\operatorname{Pr}\left\{\operatorname{Rep}\left(f s, p s, \boldsymbol{q}_{j}\right)=\emptyset \mid O_{i}=O_{j}\right\}=$ FRR.

(ii) $\operatorname{Pr}\left\{\operatorname{Rep}\left(f s, p s, \boldsymbol{q}_{j}\right)=\boldsymbol{s} \boldsymbol{s} \mid O_{i}=O_{j}\right\}=1-\mathrm{FRR}$.

(iii) $\operatorname{Pr}\left\{\operatorname{Rep}\left(f s, p s, \boldsymbol{q}_{j}\right)=\boldsymbol{s} \boldsymbol{s} \mid O_{i} \neq O_{j}\right\}=$ FAR.

(iv) $\operatorname{Pr}\left\{\operatorname{Rep}\left(f s, p s, \boldsymbol{q}_{j}\right)=\emptyset \mid O_{i} \neq O_{j}\right\}=1-$ FAR.

3) $\operatorname{Pr}\left\{\operatorname{Rep}\left(f s^{\prime}, p s^{\prime}, \boldsymbol{q}_{j}\right)=\boldsymbol{s}\right\}<\operatorname{negl}(k)$ if $f s^{\prime} \neq f s$ or $p s^{\prime} \neq f s$, where negl( $(\cdot)$ is a negligible function.

Theorem 1 (Strengthened uncoupled fuzzy extractor from a model-based uncoupling construction, an error correcting code, a universal one-way hash function and a perfectly secure symmetric encryption scheme). Let

- (KeyGen, ENC, DEC) be a $(\mathcal{F S}, \mathcal{P} \times \mathcal{H} \times \mathcal{W}, \mathcal{P S})$ perfectly secure symmetric encryption scheme,

- (ECC-enc, ECC-dec) be a $(C, n, k, t)$-error correcting code,

- $\quad(\mathrm{ME}, \mathrm{PM})$ be a $(Q, \mathcal{M}, \mathcal{P}, C, V, n)$-modelbased uncoupling construction with an associated ver $_{\mathrm{ME}, \mathrm{PM}}(\mathcal{Q}, \mathcal{M}, \mathcal{P}, C, V, n, t, \mathrm{FAR}, \mathrm{FRR})$-uncoupled model-based verifier,

- and $\mathcal{W}$ be a family of universal one-way hash functions. Let $H \subset \mathcal{W}$ be the collection of functions in $\mathcal{W}$ from $C^{k}$ to $\mathcal{H}$, which is the range of $H$.

Then, the procedures:

- Gen : $\mathcal{F S} \times \mathcal{M} \rightarrow \mathcal{P S} \times C^{k}$, which takes as input a fixed secret $f_{s}$ and a model $m_{i}$ of an individual $O_{i}$, samples $\boldsymbol{s} \boldsymbol{s} \leftarrow \mathrm{U}_{C^{k}}$ and $h \leftarrow \mathrm{U}_{H}$, and computes $\delta=$ $h(\boldsymbol{s} \boldsymbol{s}), \boldsymbol{c}=\mathrm{ECC}$-enc $(\boldsymbol{s} \boldsymbol{s}), p_{m_{i}, \boldsymbol{c}}=\mathrm{ME}\left(m_{i}, \boldsymbol{c}\right)$, and $p s=$ $\mathrm{ENC}_{f s}\left(p_{m_{i}, c}\|\delta\| h\right)$, where $\|$ stands for concatenation, and outputs $(p s, \boldsymbol{s} \boldsymbol{s})$; and

- Rep : $\mathcal{F S} \times \mathcal{P S} \times Q^{V} \rightarrow C^{k}$, which takes as input the fixed secret $f_{s}$, the public string $p s$, and a vector $\boldsymbol{q}_{j}=\left[q_{j}^{1}, \ldots, q_{j}^{V}\right] \in Q^{V}$ of samples from $Q_{j}=$ measure $\left(O_{j}\right)$, with $O_{j}$ an individual from population $O$, computes $p_{m_{i}, \boldsymbol{c}}\|\delta\| h=\operatorname{DEC}_{f s}(p s), \tilde{\boldsymbol{c}}=$ $\operatorname{PM}\left(\boldsymbol{q}_{j}, p_{m_{i}, \boldsymbol{c}}\right), \widetilde{\boldsymbol{s} s}=\operatorname{ECC}-\operatorname{dec}(\tilde{\boldsymbol{c}})$, and $\tilde{\delta}=h(\widetilde{\boldsymbol{s}})$, and outputs $\widetilde{\boldsymbol{S} S}$ if $\delta=\tilde{\delta}, \emptyset$ otherwise;

constitute an $\left(\mathcal{F S}, Q, \mathcal{M}, \mathcal{P S}, C, V, n, k, \mathrm{FAR}^{\prime}, \mathrm{FRR}^{\prime}\right)$ uncoupled extractor, with $\mathrm{FRR}^{\prime} \in(\mathrm{FRR}-\operatorname{negl}(k), \mathrm{FRR}]$ and FAR $^{\prime} \in[$ FAR, FAR $+\operatorname{negl}(k))$.

The proof of this theorem follows from the definitions of the used constructions.

The strengthened uncoupled fuzzy extractor presented in Definition III.5, and instantiated using symmetric encryption, error correcting codes, universal one-way hash functions, and model-based uncoupling constructions in Theorem 1, is illustrated in Fig. 1. The Gen procedure uses a secret key $f s$ 


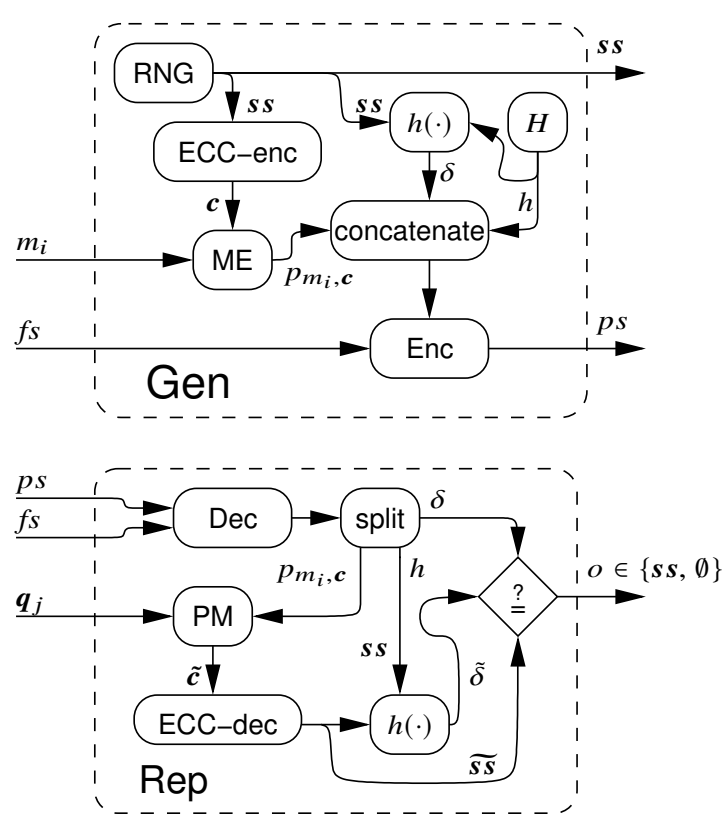

Fig. 1. Gen and Rep procedures of a strengthened uncoupled fuzzy extractor from a model-based uncoupling construction, an error correcting code, an universal one-way hash function and a perfectly secure symmetric encryption scheme.

and a template $m_{i}$ of a noisy source $O_{i}$ as input, and outputs a secret string $\boldsymbol{s} \boldsymbol{s}$ and a public string $p s$. The secret string is randomly generated, and used to encode a codeword $c$. This codeword and the template are the inputs to the PE procedure of the model-based uncoupling procedure, which computes the metadata $p_{m_{i}, \boldsymbol{c}}$. The randomly chosen hash descriptor $h$, the hash of the secret string $\delta=h(s \boldsymbol{s})$, and the metadata are concatenated and encrypted using the key $f s$ into the public string $p s$. The Rep procedure uses the public string, the secret key, and a collection of samples $\boldsymbol{q}_{j}$ from the noisy source $O_{j}$ as inputs, and outputs the secret string $\boldsymbol{s} \boldsymbol{s}$ only when the noisy sources $O_{i}$ and $O_{j}$ match. In this procedure, the public string is decrypted, obtaining the metadata, the hash descriptor, and the hash of the secret string. The metadata and the samples from the noisy source are input to the PM procedure of the modelbased uncoupling procedure, which outputs an estimate of the codeword $\tilde{\boldsymbol{c}}$. This estimate is decoded into the estimate of the secret string $\widetilde{\boldsymbol{s}}$, and the hash of this estimate is compared to the hash of the actual secret string in order to release the secret string.

This construction can be understood as an adaptation of the FE aimed at introducing a second authentication factor, a fixed (non-variable) secret key. This key can be a knowledge- or possession-based authentication factor, and it protects the metadata that drives the parametrized mapping of the noisy authentication factor in the model-based uncoupling construction. In the case that the fixed authentication factor is compromised, the adversary may only access the mapping metadata, in contrast to any key agreement protocol based on pre-existing shared secrets, where the security would be totally compromised. This metadata discloses some information about the noisy authentication factor, that can be used in some cases for linking the noisy authentication factor through different services, and therefore it has to be protected. However, this disclosure would not pose a threat to security, since the target codeword and the metadata cannot be linked, unlike in the original FE. Most important, the public and secret strings produced by this construction are perfectly uncoupled from the two authentication factors used (mutual information is 0 ), and it is trivial to produce unlinkable public and secret strings by simply changing the key, which is an additional advantage with respect to the original FE.

The role of the metadata in the model-based uncoupling construction is two-fold:

1) It allows to uncouple the target codeword from the noisy source, allowing to produce diverse secret strings for a given noisy source.

2) It allows to improve the reliability of the parametrized mapping, resulting in an improved FAR and FRR of the construction, and therefore in higher entropies of the secret string.

The first aspect is straightforward from the definitions, since the codeword is independent from the the noisy source. However, the second is not obvious, since it conveys designing a robust quantization of the noisy source. In the following sections we will show how to design an uncoupled model-based classifier for a common family of noisy sources, achieving both goals simultaneously.

\section{EFFICIENT MODEL-BASED UNCOUPLING CONSTRUCTION FOR GAUSSIAN SOURCES}

One widely used feature representation is based on the projection of the original features into an eigenspace. This kind of representation provides several advantages, including feature dimensionality reduction, denoising and decorrelation of the projected features. This approach has been used in different biometric modalities. For instance, in the case of face biometrics, some examples are the eigenface approach [21], or [22]. Eigenspace techniques have also been successfully applied to characterize temporal sequences, such as speech or online signatures. In the case of speaker recognition, its use is even more prevalent, and approaches such as the eigenvoices [23], joint factor analysis [24] or i-vectors [25] which also rely on an eigenspace transformation, are the core methods of the state-of-the-art. A similar approach has been also explored in [7] for characterizing online signature templates. These Gaussian features can be defined for the case of single and multiple sources as follows:

- Single Gaussian source: A single Gaussian source (or feature) is a measurement $Q_{i} \sim \mathcal{N}\left(\mu, \sigma^{2}\right)$ from an individual $O_{i}$ in a population $O$. This population determines the a priori distribution of the mean of the Gaussian sources. For simplicity but without loss of generality, let the Gaussian sources within the population $O$ be normalized, making the a priori distribution of the mean to be a standard Gaussian, i.e. $\mu \sim \mathcal{N}(0,1)$.

- Multiple Gaussian sources: Multiple Gaussian sources are defined as a set of independent Gaussian sources or features $\boldsymbol{Q}_{i}=\left\{Q_{i}^{1}, \ldots, Q_{i}^{F}\right\}$ characterizing individual $O_{i}$ 
in a population $O$, where $F$ is the number of features in the set. Each feature follows a Gaussian distribution, i.e.: $Q_{i}^{f} \sim \mathcal{N}\left(\mu_{f}, \sigma_{f}^{2}\right)$, and we also assume the a priori distribution of the mean to be a standard Gaussian. The independence assumption is justified by the common possibility of using feature transformations which provide uncorrelated features from jointly Gaussian features, as explained above.

We will focus on Gaussian sources with known standard deviations, but with means estimated from $E$ enrolment samples. We will only consider a model-based classifier which uses a single sample $V=1$ in the verification phase, which is the most realistic case. Also, we will constrain ourselves to the most widely used binary alphabet $C=\{0,1\}$, although equivalent constructions can be easily derived for other alphabets.

In order to construct an efficient uncoupled model-based verifier, we need a model-based uncoupling construction that aims at minimizing both FAR and FRR, defined as:

$$
\begin{aligned}
& \mathrm{FAR}=\operatorname{Pr}\left\{\mathrm{d}\left(\boldsymbol{c}, \operatorname{PM}\left(\boldsymbol{q}_{j}, \mathrm{ME}\left(m_{i}, \boldsymbol{c}\right)\right)\right) \leq t \mid O_{i} \neq O_{j}\right\} \\
& \mathrm{FRR}=\operatorname{Pr}\left\{\mathrm{d}\left(\boldsymbol{c}, \operatorname{PM}\left(\boldsymbol{q}_{j}, \operatorname{ME}\left(m_{i}, \boldsymbol{c}\right)\right)\right)>t \mid O_{i}=O_{j}\right\}
\end{aligned}
$$

Minimizing the FAR can be achieved by ensuring that $\operatorname{PM}\left(\boldsymbol{q}_{j}, \operatorname{ME}\left(m_{i}, \boldsymbol{c}\right)\right) \quad \mid O_{i} \neq O_{j} \sim \mathcal{U}_{C^{n}}$, thus providing FAR $=\sum_{\ell=0}^{t}\left(\begin{array}{l}n \\ \ell\end{array}\right)(|C|-1)^{\ell} /|C|^{n}$. On the other hand, minimizing the FRR for a given Gaussian source $O_{i}$ is equivalent to minimizing the expected Bit Error Rate $(\overline{\mathrm{BER}})$ for the genuine case $\left(O_{i}=O_{j}\right)$, defined as:

$$
\overline{\mathrm{BER}}=\mathrm{E}\left\{\frac{\mathrm{d}\left(C, \operatorname{PM}\left(Q_{i}, \operatorname{ME}\left(M_{i}, C\right)\right)\right)}{n}\right\},
$$

where $\mathrm{E}\{\cdot\}$ stands for expected value. Finally, we must keep in mind that the metadata $P_{M_{i}, C}=\operatorname{ME}\left(M_{i}, C\right)$ cannot disclose information about the discrete representation $C$, which is equivalent to stating that the probability density function (pdf) of $P_{M_{i}, C} \mid C$ is equal for all the possible values of $C$. These three conditions, i.e. (a) the minimization of the FAR, (b) the minimization of the $\overline{\mathrm{BER}}$ for genuine matches, and (c) the absence of mutual information between the discrete representation and the metadata, will drive the design of the proposed model-based uncoupling construction. We will distinguish two different cases. The first is devoted to obtain as many reliable bits, i.e., with associated $\overline{\mathrm{BER}} \leq \mathrm{BER}_{\max }$, from a single Gaussian source. Our second case is devoted to obtain a single reliable bit from a set of Gaussian sources, which is useful when these features cannot achieve a $\overline{B E R} \leq B R_{\max }$. These two derivations will allow us to design a general robust model-based uncoupling construction.

\section{A. Efficient model-based uncoupling construction for a single Gaussian source}

Let $m=\{\hat{\mu}, \sigma, E\}$ be the template of a single Gaussian source, defined in terms of a known standard deviation $\sigma$, the Maximum Likelihood estimate of the mean $\hat{\mu}$, and the number of enrolment samples $E$ used to obtain this estimate.

The optimal zero-leakage helper data, defined in [2], is denoted as quantile helper data. For fuzzy extraction constructions, they propose to use uniform quantization, and the value of the conditional cumulative distribution function (ccdf) of the mean estimate as helper data. This can be incorporated into a model-based uncoupling construction where the ME and PM functions are defined as follows:

$$
\begin{aligned}
\operatorname{ME}(m, \boldsymbol{c}) & =F_{\hat{\mu} \mid \sigma, E, \hat{\mu} \in \operatorname{bin}(\boldsymbol{c})}(\hat{\mu}) \\
\operatorname{PM}\left(q, p_{m, \boldsymbol{c}}\right) & =\underset{\tilde{\boldsymbol{c}}}{\operatorname{argmax}}\left\{f_{q \mid p_{m, \boldsymbol{c}}, \sigma, E, \hat{\mu} \in \operatorname{bin}(\tilde{\boldsymbol{c}})}(q)\right\},
\end{aligned}
$$

where $F_{\hat{\mu} \mid \sigma, E, \hat{\mu} \in \operatorname{bin}(\boldsymbol{c})}(\hat{\mu})$ is the ccdf of the mean estimate in the uniform quantization case, and $f_{q \mid p_{m, \boldsymbol{c}}, \sigma, E, \hat{\mu} \in \operatorname{bin}(\boldsymbol{c})}(q)$ is the conditional pdf of the verification sample $q$, given that the metadata is $p_{m, c}$, and in both cases given that the mean estimated from $E$ enrollment samples is $\hat{\mu} \in \operatorname{bin}(\boldsymbol{c})$. Zero-leakage approaches are defined by using any continuous monotonic function of the cumulative distribution function. We will theoretically compare this approach with the baseline case, where uniform quantization is used, but no helper data is incorporated.

Gain in False Rejection Rate of the zero-leakage approach: In order to evaluate the gain provided by the use of the helper data in the zero-leakage helper data model-based uncoupling construction, we will evaluate the $\overline{\mathrm{BER}}$ for the genuine case in our construction, denoted as $\overline{\mathrm{BER}}_{n \text {-bit }}^{\text {-leakage }}(\sigma, E)$, and we will compare it with the average BER in the baseline case, denoted as $\overline{\mathrm{BER}}_{n \text {-bit }}^{\text {baseline }}(\sigma, E)$.

Let us denote the set of thresholds defining the uniform quantization bins as $\Theta=\left\{\theta_{i}, i \in\left\{0, \ldots, 2^{n}\right\}\right\}$, where:

$$
\theta_{i}=\sqrt{1+\frac{\sigma^{2}}{E}} \operatorname{erfinv}\left(\frac{i-2^{n-1}}{2^{n-1}}\right) .
$$

Let us denote the cumulative distribution function of the genuine sample $q \sim \mathcal{N}\left(\mu, \sigma^{2}\right)$ as $F_{q}(q)=\frac{1}{2}\left[1+\operatorname{erf}\left(\frac{q-\mu}{\sigma \sqrt{2}}\right)\right]$. Let us also denote the $n$-bit Gray encoding of the $i$-th bin as $G_{i}^{n}$, and $\tau_{i}(\hat{\mu})=F_{\hat{\mu} \mid \sigma, E, \hat{\mu} \in \operatorname{bin}\left(G_{i}^{n}\right)}(\hat{\mu})$, defined as:

$$
\tau_{i}(\hat{\mu})=\frac{\operatorname{erf}\left(\hat{\mu} / \sqrt{2\left(1+\frac{\sigma^{2}}{E}\right)}\right)-\operatorname{erf}\left(\theta_{i} / \sqrt{2\left(1+\frac{\sigma^{2}}{E}\right)}\right)}{\operatorname{erf}\left(\theta_{i+1} / \sqrt{2\left(1+\frac{\sigma^{2}}{E}\right)}\right)-\operatorname{erf}\left(\theta_{i} / \sqrt{2\left(1+\frac{\sigma^{2}}{E}\right)}\right)} .
$$

Finally, let us denote the value of the estimated mean in the bin $j$ that would produce $\tau=\tau_{j}(\hat{\mu})=\tau_{i}(\hat{\mu})$ as:

$$
\begin{aligned}
& \tilde{\mu}_{j}(\tau)=F_{\hat{\mu} \mid \sigma, E, \hat{\mu} \in \operatorname{bin}\left(G_{j}^{n}\right)}^{-1}(\tau)= \\
& \sqrt{2\left(1+\sigma^{2} / E\right)} \operatorname{erfinv}\left\{\tau \left[\operatorname{erf}\left(\theta_{j+1} / \sqrt{2\left(1+\sigma^{2} / E\right)}\right)-\right.\right. \\
& \left.\left.\operatorname{erf}\left(\theta_{j} / \sqrt{2\left(1+\sigma^{2} / E\right)}\right)\right]+\operatorname{erf}\left(\theta_{j} / \sqrt{2\left(1+\sigma^{2} / E\right)}\right)\right\} .
\end{aligned}
$$

Then, $\overline{\operatorname{BER}}_{n \text {-bit }}^{0 \text {-leake }}(\sigma, E)$ is shown in (15). The asymptotic case where $E=\infty$ is shown in (16), where $\tilde{\mu}_{j}(\tau)$ is defined as:

$$
\begin{aligned}
\tilde{\mu}_{j}(\tau) & =\lim _{E \rightarrow \infty} F_{\hat{\mu} \mid \sigma, E, \hat{\mu} \in \operatorname{bin}\left(G_{j}^{n}\right)}^{-1}(\tau) \\
& =\sqrt{2} \operatorname{erfinv}\left\{\tau\left[\operatorname{erf}\left(\frac{\theta_{j+1}}{\sqrt{2}}\right)-\operatorname{erf}\left(\frac{\theta_{j}}{\sqrt{2}}\right)\right]+\operatorname{erf}\left(\frac{\theta_{j}}{\sqrt{2}}\right)\right\}
\end{aligned}
$$


In the baseline case, where no helper data is used, the expression for the average BER for the genuine case, de-

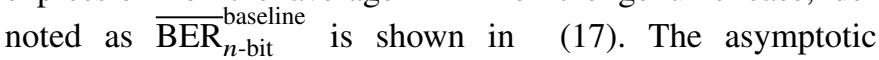
case where $E=\infty$ is shown in (18). Although there is not an analytical solution to the integral shown in this equation, it exhibits nice numerical properties, allowing to numerically approximate it. Fig. 2 shows $\overline{\operatorname{BER}}_{n \text {-bit }}^{\text {O-leake }}(\sigma, E)$ and $\overline{\mathrm{BER}}_{n \text {-bit }}^{\text {baseline }}(\sigma, E)$ for the asymptotic case. This clearly illustrates the gain provided by the zero-leakage helper data regarding $\overline{\mathrm{BER}}$ for the genuine case, which is equivalent to provide a gain in terms of False Rejection Rate. Fig. 3 focuses on the case $n=3$, showing $\overline{\mathrm{BER}}_{n \text {-bit }}^{\text {O-leakage }}(\sigma, E)$ for different number of enrolment samples, and $\overline{\operatorname{BER}}_{n \text {-bit }}^{\text {baseline }}(\sigma, E)$ for the asymptotic case. This figure illustrates the gain provided by using more samples during the enrolment when using a zeroleakage approach, but it also shows that using this helper data provides better error rates than the baseline approach even when the latter has perfect knowledge of the genuine samples distribution.

\section{B. Efficient model-based uncoupling construction for multiple unreliable Gaussian sources}

In the previous section we have focused on extracting as many bits as possible from a single Gaussian source. However, if our construction must provide $\overline{\mathrm{BER}} \leq \mathrm{BER}_{\max }$, it may be the case that for some Gaussian features $\overline{\operatorname{BER}}_{1 \text {-bit }}^{\text {-leake }}(\sigma, E)>$ $\mathrm{BER}_{\max }$. This can be inferred from Fig. 2, where it is clear that the BER grows with the Gaussian source's standard deviation.

A feature $f \sim \mathcal{N}\left(\mu, \sigma^{2}\right)$ is considered as BER max $_{\text {-unreliable }}$ for $E$ enrolment samples if the following condition holds:

$$
\sigma>\max \left\{\sigma^{\prime} \mid \overline{\operatorname{BER}}_{1 \text {-bit }}^{\text {0-leakage }}\left(\sigma^{\prime}, E\right) \leq \mathrm{BER}_{\max }\right\} .
$$

The simplest approach to deal with unreliable Gaussian sources is simply to discard them. However, this approach is not using all the available fuzzy information, which degrades the overall performance in terms of key length of the construction. In this section, we show how to optimaly combine unreliable Gausian features to obtain reliable bits.

We will focus on designing a general procedure to combine $F \mathrm{BER}_{\max }$-unreliable independent Gaussian sources $\mathcal{F}=$ $\left\{f_{1}, \ldots, f_{F}\right\}$, with $f_{i} \sim \mathcal{N}\left(\mu_{i}, \sigma_{i}^{2}\right)$ into the maximum possible number $n$ of binary sources $\mathcal{B}=\left\{b_{1}, \ldots, b_{n}\right\}$ such that $\max _{i \in\{1, \ldots, n\}}\left\{\overline{\mathrm{BER}}_{b_{i}}\right\} \leq \mathrm{BER}_{\max }$. In this case, our Gaussian sources are described by the template:

$$
m=\left\{\left(\hat{\mu}_{1}, \sigma_{1}\right), \ldots,\left(\hat{\mu}_{F}, \sigma_{F}\right), E\right\},
$$

where $E$ is the number of available enrolment samples, $\hat{\mu}_{i}$ is the estimate of the mean of the Gaussian source $f_{i}$, and $\sigma_{i}$ is the standard deviation (we assume it is known). We first describe the combination procedure for obtaining one single binary source with minimum $\overline{\mathrm{BER}}$ from two Gaussian sources, then from $F$ Gaussian sources, and then we will explain the general procedure to combine $F$ Gaussian sources into the maximum number of binary sources with a $\overline{\mathrm{BER}}$ below a certain $\mathrm{BER}_{\max }$.
1) One-bit efficient model-based uncoupling construction for two unreliable Gaussian sources: We analyze the combination of two Gaussian features $f_{1} \sim \mathcal{N}\left(\mu_{1}, \sigma_{1}^{2}\right)$ and $f_{2} \sim$ $\mathcal{N}\left(\mu_{2}, \sigma_{2}^{2}\right)$ into a new one $f=f_{1}+a f_{2} \sim \mathcal{N}\left(\mu_{1}+a \mu_{2}, \sigma_{1}^{2}+\right.$ $a^{2} \sigma_{2}^{2}$ ), when the standard deviations are known, but the means are estimated from $E$ samples $q_{1}^{i}, \ldots, q_{E}^{i}$, i.e. $\hat{\mu}_{i}=\frac{1}{E} \sum_{j=1}^{E} q_{j}^{i}$. The metadata extraction and parametrized mapping functions would in this case simply defined as:

$$
\operatorname{ME}(m, c)=a, \quad \operatorname{PM}(\boldsymbol{q}, a)=\frac{\operatorname{sign}\left(q_{1}+a q_{2}\right)+1}{2},
$$

where $m=\left\{\left(\hat{\mu}_{1}, \sigma_{1}\right),\left(\hat{\mu}_{2}, \sigma_{2}\right), E\right\}, a \in \mathbb{R}, \boldsymbol{q} \in \mathbb{R}^{2}, c \in\{0,1\}$, and $\operatorname{sign}(x)=x /|x|$ for $x \neq 0$, and $\operatorname{sign}(0)=0$. Our goal is to minimize the $\overline{\mathrm{BER}}$ when $q_{i} \sim \mathcal{N}\left(\mu_{i}, \sigma_{i}\right)$ in $(21)$, i.e., in the genuine case, where:

$$
\begin{aligned}
& \overline{\operatorname{BER}}\left(\hat{\mu}_{1}, \hat{\mu}_{2}, \sigma_{1}, \sigma_{2}, a, E\right)=\int_{\mu_{1}=-\infty}^{+\infty} \int_{\mu_{2}=-\infty}^{+\infty} \\
& \frac{1}{2}\left\{1-\operatorname{sign}\left[\left(\mu_{1}+a \mu_{2}\right)\left(\hat{\mu}_{1}+a \hat{\mu}_{2}\right)\right] \operatorname{erf}\left(\frac{\left|\mu_{1}+a \mu_{2}\right|}{\sqrt{2\left(\sigma_{1}^{2}+a^{2} \sigma_{2}^{2}\right)}}\right)\right\}
\end{aligned}
$$$$
f_{\mu_{1}, \mu_{2} \mid \hat{\mu}_{1}, \hat{\mu}_{2}}\left(\mu_{1}, \mu_{2}\right) d \mu_{2} d \mu_{1} \text {, }
$$

where:

$$
f_{\mu_{1}, \mu_{2} \mid \hat{\mu}_{1}, \hat{\mu}_{2}}\left(\mu_{1}, \mu_{2}\right)=\frac{E}{2 \pi \sigma_{1} \sigma_{2}} e^{-\frac{E\left(\mu_{1}-\hat{\mu}_{1}\right)^{2}}{2 \sigma_{1}^{2}}} e^{-\frac{E\left(\mu_{2}-\hat{\mu}_{2}\right)^{2}}{2 \sigma_{2}^{2}}} .
$$

This equation provides also the solution to the known $\mu$ case:

$$
\begin{aligned}
& \overline{\operatorname{BER}}\left(\mu_{1}, \mu_{2}, \sigma_{1}, \sigma_{2}, a, \infty\right)=\lim _{E \rightarrow \infty} \overline{\operatorname{BER}}\left(\hat{\mu}_{1}, \hat{\mu}_{2}, \sigma_{1}, \sigma_{2}, a, E\right) \\
& =\frac{1}{2}\left[1-\operatorname{erf}\left(\frac{\left|\mu_{1}+a \mu_{2}\right|}{\sqrt{2} \sqrt{\sigma_{1}^{2}+a^{2} \sigma_{2}^{2}}}\right)\right]=\frac{1}{2}\left[1-\operatorname{erf}\left(\frac{|\rho|}{\sqrt{2}}\right)\right],
\end{aligned}
$$

where $\rho=\left(\mu_{1}+a \mu_{2}\right) / \sqrt{\sigma_{1}^{2}+a^{2} \sigma_{2}^{2}}$. If for any Gaussian variable $\star \sim \mathcal{N}\left(\mu_{\star}, \sigma_{\star}^{2}\right)$ we define $\rho_{\star}=\mu_{\star} / \sigma_{\star}$, and its estimate as $\hat{\rho}_{\star}=\hat{\mu}_{\star} / \sigma_{\star}$, and $s=\frac{\sigma_{1}}{\sigma_{2}}$, then Equation (22) can be expressed as:

$$
\begin{aligned}
& \overline{\operatorname{BER}}\left(\hat{\rho}_{1}, \hat{\rho}_{2}, s, a, E\right)=\int_{\rho_{1}=-\infty}^{+\infty} \int_{\rho_{2}=-\infty}^{+\infty} \\
& \frac{1}{2}\left\{1-\operatorname{sign}\left[\left(s \rho_{1}+a \rho_{2}\right)\left(s \hat{\rho}_{1}+a \hat{\rho}_{2}\right)\right] \operatorname{erf}\left(\frac{\left|s \rho_{1}+a \rho_{2}\right|}{\sqrt{2\left(s^{2}+a^{2}\right)}}\right)\right\} \\
& f_{\rho_{1}, \rho_{2} \mid \hat{\rho}_{1}, \hat{\rho}_{2}}\left(\rho_{1}, \rho_{2}\right) d \rho_{2} d \rho_{1},
\end{aligned}
$$

where:

$$
f_{\rho_{1}, \rho_{2} \mid \hat{\rho}_{1}, \hat{\rho}_{2}}\left(\rho_{1}, \rho_{2}\right)=\frac{E}{2 \pi} e^{-\frac{E\left(\rho_{1}-\hat{\rho}_{1}\right)^{2}}{2}} e^{-\frac{E\left(\rho_{2}-\hat{\rho}_{2}\right)^{2}}{2}} .
$$

It must be noted that the optimal value of $a$ is independent of $E$, and it is equal to:

$$
a_{\text {opt }}=\underset{a}{\operatorname{argmin}}\left\{\overline{\operatorname{BER}}\left(\hat{\rho}_{1}, \hat{\rho}_{2}, s, a, E\right)\right\}=s \frac{\hat{\rho}_{2}}{\hat{\rho}_{1}} .
$$

However, the BER is bigger when the uncertainty on the value of $\mu_{1}$ and $\mu_{2}$ is high (small values of $E$ ), as it can be observed 


$$
\begin{aligned}
& \overline{\mathrm{BER}}_{n \text {-bit }}^{\text {O-leakage }}(\sigma, E)=\mathrm{E}\left\{\mathrm{BER}_{n \text {-bit }}^{0 \text {-leakage }}(\mu, \hat{\mu}, \sigma, E)\right\} \\
& =\sum_{i=0}^{2^{n}-1} \sum_{j=0}^{2^{n}-1} \frac{d_{\mathcal{H}}\left(G_{i}^{n}, G_{j}^{n}\right)}{n} \operatorname{Pr}\left(\hat{\mu} \in\left[\theta_{i}, \theta_{i+1}\right), q \in\left[\frac{\tilde{\mu}_{j-1}\left(\tau_{i}(\hat{\mu})\right)+\tilde{\mu}_{j}\left(\tau_{i}(\hat{\mu})\right)}{2}, \frac{\tilde{\mu}_{j}\left(\tau_{i}(\hat{\mu})\right)+\tilde{\mu}_{j+1}\left(\tau_{i}(\hat{\mu})\right)}{2}\right) \mid \begin{array}{l}
q \sim \mathcal{N}\left(\mu, \sigma^{2}\right) \\
\mu \sim \mathcal{N}(0,1)
\end{array}, \hat{\mu} \sim \mathcal{N}\left(\mu, \frac{\sigma^{2}}{E}\right)\right) \\
& =\frac{\sqrt{E}}{2 n \pi \sigma} \sum_{i=0}^{2^{n}-1} \sum_{j=0}^{2^{n}-1} d_{\mathcal{H}}\left(G_{i}^{n}, G_{j}^{n}\right) \int_{\mu=-\infty}^{\mu=+\infty} \int_{\hat{\mu}=\theta_{i}}^{\hat{\mu}=\theta_{i+1}}\left[F_{q}\left(\frac{\tilde{\mu}_{j}\left(\tau_{i}(\hat{\mu})\right)+\tilde{\mu}_{j+1}\left(\tau_{i}(\hat{\mu})\right)}{2}\right)-F_{q}\left(\frac{\tilde{\mu}_{j-1}\left(\tau_{i}(\hat{\mu})\right)+\tilde{\mu}_{j}\left(\tau_{i}(\hat{\mu})\right)}{2}\right)\right] e^{-\frac{E(\mu-\hat{\mu})^{2}}{2 \sigma^{2}}} \mathrm{~d} \hat{\mu} e^{-\frac{\mu^{2}}{2}} \mathrm{~d} \mu \\
& \overline{\operatorname{BER}}_{n \text {-bit }}^{0 \text {-leakage }}(\sigma, \infty)=\lim _{E \rightarrow \infty}\left\{\mathrm{E}\left\{\operatorname{BER}_{n \text {-bit }}^{0 \text {-leakage }}(\mu, \hat{\mu}, \sigma, E)\right\}\right\} \\
& =\sum_{i=0}^{2^{n}-1} \sum_{j=0}^{2^{n}-1} \frac{d_{\mathcal{H}}\left(G_{i}^{n}, G_{j}^{n}\right)}{n} \operatorname{Pr}\left(\mu \in\left[\theta_{i}, \theta_{i+1}\right), q \in\left[\frac{\tilde{\mu}_{j-1}\left(\tau_{i}(\hat{\mu})\right)+\tilde{\mu}_{j}\left(\tau_{i}(\hat{\mu})\right)}{2}, \frac{\tilde{\mu}_{j}\left(\tau_{i}(\hat{\mu})\right)+\tilde{\mu}_{j+1}\left(\tau_{i}(\hat{\mu})\right)}{2}\right) \mid \begin{array}{l}
q \sim \mathcal{N}\left(\mu, \sigma^{2}\right) \\
\mu \sim \mathcal{N}(0,1)
\end{array}\right) \\
& =\frac{1}{n \sqrt{2 \pi}} \sum_{i=0}^{2^{n}-1} \sum_{j=0}^{2^{n}-1} d_{\mathcal{H}}\left(G_{i}^{n}, G_{j}^{n}\right) \int_{\mu=\theta_{i}}^{\mu=\theta_{i+1}}\left[F_{q}\left(\frac{\tilde{\mu}_{j}\left(\tau_{i}(\hat{\mu})\right)+\tilde{\mu}_{j+1}\left(\tau_{i}(\hat{\mu})\right)}{2}\right)-F_{q}\left(\frac{\tilde{\mu}_{j-1}\left(\tau_{i}(\hat{\mu})\right)+\tilde{\mu}_{j}\left(\tau_{i}(\hat{\mu})\right)}{2}\right)\right] e^{-\frac{\mu^{2}}{2}} \mathrm{~d} \mu \\
& \overline{\operatorname{BER}}_{n \text {-bit }}^{\text {baseline }}(\sigma, E)=\mathrm{E}\left\{\operatorname{BER}_{n \text {-bit }}^{\text {baseline }}(\mu, \hat{\mu}, \sigma, E)\right\} \\
& =\sum_{i=0}^{2^{n}-1} \sum_{j=0}^{2^{n}-1} \frac{d_{\mathcal{H}}\left(G_{i}^{n}, G_{j}^{n}\right)}{n} \operatorname{Pr}\left(\hat{\mu} \in\left[\theta_{i}, \theta_{i+1}\right), q \in\left[\theta_{j}, \theta_{j+1}\right) \mid \begin{array}{l}
q \sim \mathcal{N}\left(\mu, \sigma^{2}\right) \\
\mu \sim \mathcal{N}(0,1)
\end{array}, \hat{\mu} \sim \mathcal{N}\left(\mu, \frac{\sigma^{2}}{E}\right)\right) \\
& =\frac{\sqrt{E}}{2 n \pi \sigma} \sum_{i=0}^{2^{n}-1} \sum_{j=0}^{2^{n}-1} d_{\mathcal{H}}\left(G_{i}^{n}, G_{j}^{n}\right) \int_{\mu=-\infty}^{\mu=+\infty} \int_{\hat{\mu}=\theta_{i}}^{\hat{\mu}=\theta_{i+1}}\left[F_{q}\left(\theta_{j+1}\right)-F_{q}\left(\theta_{j}\right)\right] e^{-\frac{E(\mu-\hat{\mu})^{2}}{2 \sigma^{2}}} \mathrm{~d} \hat{\mu} e^{-\frac{\mu^{2}}{2}} \mathrm{~d} \mu \\
& \overline{\mathrm{BER}}_{n \text {-bit }}^{\text {baseline }}(\sigma, \infty)=\lim _{E \rightarrow \infty} \mathrm{E}\left\{\mathrm{BER}_{n \text {-bit }}^{\text {baseline }}(\mu, \hat{\mu}, \sigma, E)\right\} \\
& =\sum_{i=0}^{2^{n}-1} \sum_{j=0}^{2^{n}-1} \frac{d_{\mathcal{H}}\left(G_{i}^{n}, G_{j}^{n}\right)}{n} \operatorname{Pr}\left(\mu \in\left[\theta_{i}, \theta_{i+1}\right), q \in\left[\theta_{j}, \theta_{j+1}\right) \mid \begin{array}{l}
q \sim \mathcal{N}\left(\mu, \sigma^{2}\right) \\
\mu \sim \mathcal{N}(0,1)
\end{array}\right) \\
& =\frac{1}{n \sqrt{2 \pi}} \sum_{i=0}^{2^{n}-1} \sum_{j=0}^{2^{n}-1} d_{\mathcal{H}}\left(G_{i}^{n}, G_{j}^{n}\right) \int_{\mu=\theta_{i}}^{\mu=\theta_{i+1}}\left[F_{q}\left(\theta_{j+1}\right)-F_{q}\left(\theta_{j}\right)\right] e^{-\frac{\mu^{2}}{2}} \mathrm{~d} \mu
\end{aligned}
$$

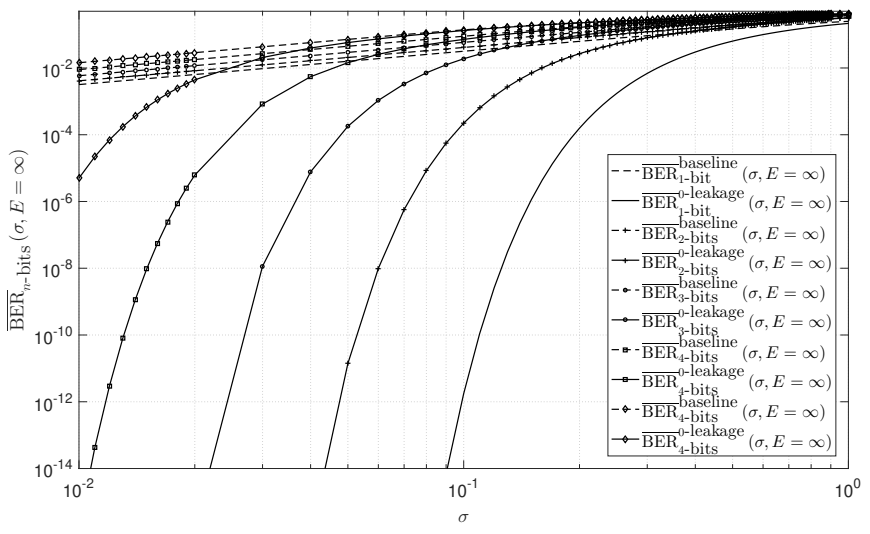

Fig. 2. $\overline{\operatorname{BER}}_{n \text {-bit }}^{\text {baseline }}(\sigma, E)$ and $\overline{\mathrm{BER}}_{n \text {-bit }}^{0 \text {-leake }}(\sigma, E)$ for the asymptotic case $E=\infty$ as a function of the standard deviation of the genuine distribution $\sigma$.

in Fig. 4. Also, when $a=a_{\text {opt }}$ we obtain the following rotationinvariant expression, independent of $s$ :

$$
\begin{aligned}
& \overline{\operatorname{BER}}_{\text {opt }}\left(\hat{\rho}_{1}, \hat{\rho}_{2}, E\right)= \\
& \int_{\rho_{1}=-\infty}^{+\infty} \int_{\rho_{2}=-\infty}^{+\infty} \frac{1}{2}\left[1-\operatorname{erf}\left(\frac{\rho_{1} \hat{\rho}_{1}+\rho_{2} \hat{\rho}_{2}}{\sqrt{2\left(\hat{\rho}_{1}^{2}+\hat{\rho}_{2}^{2}\right)}}\right)\right] \\
& \frac{E}{2 \pi} e^{-\frac{E\left(\rho_{1}-\hat{\rho}_{1}\right)^{2}}{2}} e^{-\frac{E\left(\rho_{2}-\hat{\rho}_{2}\right)^{2}}{2}} d \rho_{2} d \rho_{1} .
\end{aligned}
$$

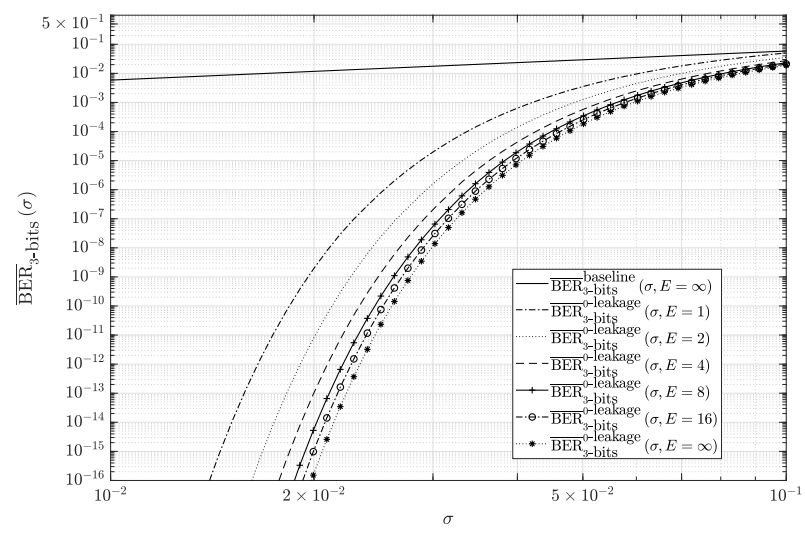

Fig. 3. $\overline{\mathrm{BER}}_{\text {3-bits }}^{\text {baseline }}(\sigma, E=\infty)$ and $\overline{\mathrm{BER}}_{\text {3-bits }}^{\text {O-leakage }}(\sigma, E)$ for $E \quad \in$ $\{1,2,4,8,16, \infty\}$ as a function of the standard deviation of the genuine distribution $\sigma$.

This rotation invariance allows us to simplify this expression in terms of a more simple integral. Let $\hat{\rho}=\sqrt{\hat{\rho}_{1}^{2}+\hat{\rho}_{2}^{2}}$. Then the $\overline{\mathrm{BER}}_{\mathrm{opt}}$ can be calculated as:

$$
\overline{\mathrm{BER}}_{\mathrm{opt}}(\hat{\rho}, E)=\int_{\rho=-\infty}^{+\infty} \frac{1}{2}\left[1-\operatorname{erf}\left(\frac{\hat{\rho}}{\sqrt{2}}\right)\right] \frac{\sqrt{E}}{\sqrt{2 \pi}} e^{-\frac{E(\rho-\hat{\rho})^{2}}{2}} d \rho .
$$




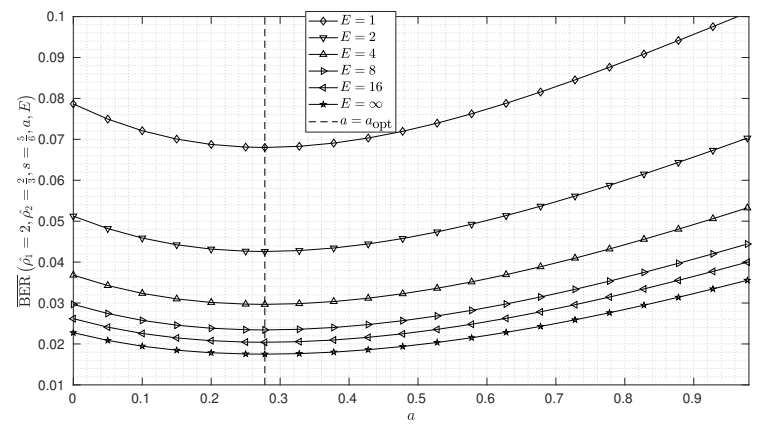

Fig. 4. $\overline{\text { BER }}$ for different values of $E$ and a given setup $\left(\hat{\rho}_{1}=2, \hat{\rho}_{2}=\frac{2}{3}, s=\right.$ $\left.\frac{5}{6}, a_{\mathrm{opt}}=\frac{5}{18}\right)$.

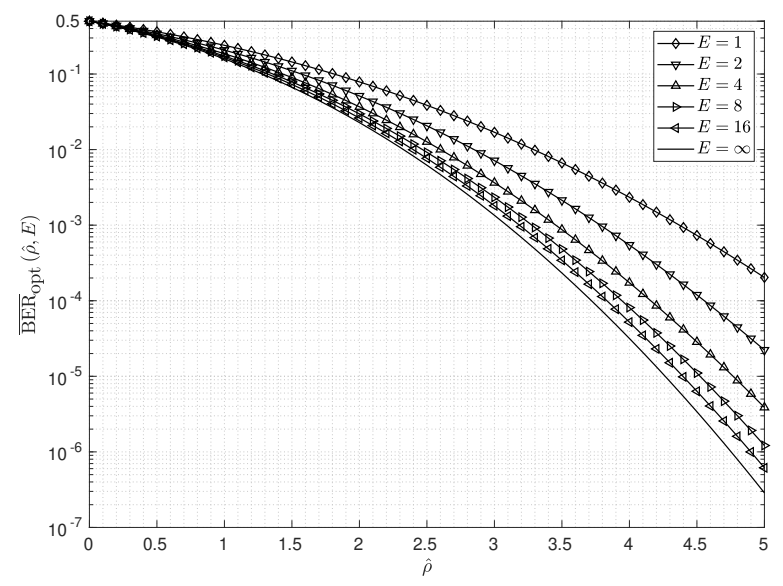

Fig. 5. $\overline{\mathrm{BER}}_{\mathrm{opt}}(\hat{\rho}, E)$ for different values of $E$ versus the estimated reliability of the combined Gaussian feature $\hat{\rho}$

In general, for a combination of $F$ features with estimated reliabilities $\hat{\rho}_{1}=\hat{\mu}_{1} / \sigma_{1}, \ldots, \hat{\rho}_{F}=\hat{\mu}_{F} / \sigma_{F}$, if we define the squared global estimated reliability as $\hat{\rho}^{2}=\sum_{i=1}^{F} \hat{\rho}_{i}^{2}$ then $\overline{\mathrm{BER}}_{\mathrm{opt}}$ can be calculated using (29). Fig. 5 shows $\overline{\mathrm{BER}}_{\mathrm{opt}}$ versus the estimated reliability $\hat{\rho}$ for different values of $E$, including the asymptotic case $E=\infty$.

2) One-bit efficient model-based uncoupling construction for multiple unreliable Gaussian sources: We are interested in obtaining the model-based uncoupling construction consisting of the following metadata extraction and parametrized mapping functions:

$$
\begin{aligned}
\operatorname{ME}\left(m_{f}, c\right) & =\boldsymbol{w}, \\
\operatorname{PM}(\boldsymbol{q}, \boldsymbol{w}) & =\frac{\operatorname{sign}\left(\boldsymbol{w}^{t} \boldsymbol{q}\right)+1}{2},
\end{aligned}
$$

where $\boldsymbol{q}=\left[q_{1}, \ldots, q_{F}\right]^{t} \in \mathbb{R}^{F}$, with $m_{\boldsymbol{f}}$ defined as in (20), and such that the $\overline{\mathrm{BER}}$ is minimum when $q_{i} \sim \mathcal{N}\left(\mu_{i}, \sigma_{i}^{2}\right)$. In order to achieve this goal, we sort the Gaussian sources according to $\left|\hat{\rho}_{i}\right|=\left|\frac{\hat{\mu}_{i}}{\sigma_{i}}\right|$, obtaining $\mathcal{F}_{\text {sorted }}=\left\{f_{i_{1}}, \ldots, f_{i_{F}}\right\}$, such that $\left|\hat{\rho}_{i_{1}}\right| \geq\left|\hat{\rho}_{i_{2}}\right| \geq \cdots \geq\left|\hat{\rho}_{i_{F}}\right|$. The combined feature is defined as $g=\sum_{j=1}^{F} w_{i_{j}} f_{i_{j}}$, where the components of the weights vector $\mathbf{w}=\left[w_{1}, \ldots, w_{F}\right]^{t}$ are defined as:

$$
w_{i_{k}}= \begin{cases}\operatorname{sign}(2 c-1) \operatorname{sign}\left(\hat{\mu}_{i_{1}}\right) & , \text { for } k=1, \\ \frac{\hat{\rho}_{i_{k}} \operatorname{sign}\left(w_{i_{1}} \hat{\rho}_{i_{1}}\right) \sqrt{\sum_{j=1}^{k-1} w_{i_{j}}^{2} \sigma_{i_{j}}^{2}}}{\sigma_{i_{k}} \sqrt{\sum_{j=1}^{k-1} \hat{\rho}_{i_{j}}^{2}}} & , \text { for } k>1 .\end{cases}
$$

With the weight vectors chosen as above, the reliability of the combined feature is the optimal one, i.e. $\hat{\rho}_{g}^{2}=\sum_{i=1}^{F} \hat{\rho}_{i}^{2}$, and the $\overline{\mathrm{BER}}$ can be computed using (29).

3) General case for unreliable Gaussian features: Multiplebit efficient model-based uncoupling construction for multiple unreliable Gaussian sources: Here we will focus on the case where a set of $\mathrm{BER}_{\max }$-unreliable features must provide the maximum possible number of bits $b_{i}$ such that their associated average BER is $\overline{\operatorname{BER}}\left(b_{i}\right) \leq \mathrm{BER}_{\max }$ in the genuine case. The corresponding metadata extraction and parametrized mapping functions can be defined as:

$$
\begin{aligned}
\operatorname{ME}\left(m_{f}, \boldsymbol{c}\right) & =\left(\left\{\mathcal{A}_{1}, \ldots, \mathcal{A}_{n}\right\}, \boldsymbol{w}\right), \\
\operatorname{PM}(\boldsymbol{q}, \boldsymbol{w}) & =\left[\begin{array}{c}
\frac{\operatorname{sign}\left(\sum_{i \in \mathcal{A}_{1}} w_{i} q_{i}\right)+1}{2} \\
\vdots \\
\frac{\operatorname{sign}\left(\sum_{i \in \mathcal{A}_{n}} w_{i} q_{i}\right)+1}{2}
\end{array}\right] .
\end{aligned}
$$

We need that each of the combined features $g_{\mathcal{A}_{i}}$ are reliable enough, i.e. $\hat{\rho}_{\mathcal{A}_{i}}^{2} \geq\left[\overline{\mathrm{BER}}_{\mathrm{opt}}^{-1}\left(\mathrm{BER}_{\max }, E\right)\right]^{2}$. Therefore, the number of bits that can be allocated from a set of features $\mathcal{F}$ with $\hat{\rho}_{\mathcal{F}}^{2}=\sum_{i=1}^{F} \hat{\rho}_{i}^{2}$ is:

$$
n_{\max }\left(\hat{\rho}_{\mathcal{F}}\right) \leq\left\lfloor\frac{\hat{\rho}_{\mathcal{F}}^{2}}{\left[\overline{\mathrm{BER}}_{\mathrm{opt}}^{-1}\left(\mathrm{BER}_{\max }, E\right)\right]^{2}}\right\rfloor .
$$

Finding the optimal sets $\mathcal{A}_{i}$ is an example of the Multi-Way Number Partitioning [26]. Although this is a NP-complete problem, there are multiple approaches to tackle it, even optimal ones, as those described in [27]. However, the following Greedy approach can perform reasonably well:

1) Initialize:

a) Compute $\hat{\rho}_{\mathcal{F}}^{2}=\sum_{f_{i} \in \mathcal{F}} \hat{\rho}_{i}^{2}$.

b) Set $n=n_{\max }\left(\hat{\rho}_{\mathcal{F}}\right)$.

c) Define $\mathcal{D}=\mathcal{F}=\left\{f_{1}, \ldots, f_{F}\right\}$ as the set of features that need to be allocated.

2) Define $\mathcal{A}_{1}=\emptyset, \ldots, \mathcal{A}_{n}=\emptyset$ as the sets of features allocated to each binary source $b_{1}, \ldots, b_{n}$ respectively.

3) Input $\boldsymbol{c} \in\{0,1\}^{n}$.

4) Set iteration counter it $=1$.

5) While $i t \leq F$ do:

a) Compute $\hat{\rho}_{\mathcal{A}_{1}}, \ldots, \hat{\rho}_{\mathcal{A}_{n}}$ :

- If $\mathcal{A}_{i}=\emptyset$ then $\hat{\rho}_{\mathcal{A}_{i}}=0$.

- Otherwise, $\hat{\rho}_{\mathcal{A}_{i}}=\sum_{f_{j} \in \mathcal{A}_{i}} \hat{\rho}_{j}^{2}$

b) Let $i^{i t}=\operatorname{argmax}_{f_{i} \in \mathcal{D}} \hat{\rho}_{i}$ be the index of the most reliable Gaussian source not allocated yet.

c) Let $j^{i t}=\operatorname{argmin}_{j} \hat{\rho}_{\mathcal{A}_{j}}$ be the index of the less reliable bit.

d) If $\mathcal{A}_{j^{i t}} \neq \emptyset$ then define $w_{i^{i t}}=\frac{\hat{\rho}_{i^{i t}}}{\hat{\rho}_{\mathcal{A}_{j} i t}} \frac{\sigma_{\mathcal{A}_{j} i t}}{\sigma_{i^{i t}}}$. Else, define $w_{i^{i t}}=\operatorname{sign}(\hat{\mu}) \operatorname{sign}\left(2 c_{j^{i t}}-1\right)$. 
e) Set $\mathcal{A}_{j^{i t}}=\mathcal{A}_{j^{i t}} \cup\left\{f_{i^{i t}}\right\}$.

f) Set $\mathcal{D}=\mathcal{D}-\left\{f_{i t}\right\}$.

g) Set $i t=i t+1$.

6) If $\min _{j} \hat{\rho}_{\mathcal{A}_{j}} \geq \overline{\mathrm{BER}}_{\mathrm{opt}}^{-1}\left(\mathrm{BER}_{\max }, E\right)$, then return $\left(\left\{\mathcal{A}_{1}, \ldots, \mathcal{A}_{n}\right\}, \mathbf{w}\right)$; else set $n=n-1, \mathcal{D}=\mathcal{F}$, and go to step 2 .

\section{General efficient model-based uncoupling construction for Gaussian sources}

The constructions presented in Sect. IV-A and IV-B can be used to provide a general procedure to build a general robust model-based uncoupling construction for Gaussian sources. The starting point is a set of Gaussian sources, and the requirement is a given $\mathrm{BER}_{\max }$. The model-based uncoupling construction for Gaussian sources must encode the maximum possible number of reliable bits $n_{\max }$ with a BER below $\mathrm{BER}_{\max }$ using the robust model-based uncoupling constructions from the previous sections. First, we need to calculate the maximum possible number of reliable bits that we can extract from each single Gaussian source $Q_{i} \sim \mathcal{N}\left(\mu_{i}, \sigma_{i}^{2}\right)$ with model $m_{i}=\left(\hat{\mu}_{i}, \sigma_{i}, E\right)$, with $i \in\{1, \ldots, F\}$ :

$$
n_{i}\left(m_{i}, \mathrm{BER}_{\max }\right)=\max \left\{j \mid \overline{\operatorname{BER}}_{j \text {-bit }}^{\text {O-leakage }}\left(\sigma_{i}, E\right) \leq \mathrm{BER}_{\max }\right\},
$$

where for convenience we set $\overline{\mathrm{BER}}_{0 \text {-bit }}^{0 \text {-leage }}\left(\sigma_{i}, E\right)=0$. Let us define the set of the indexes of the $\mathrm{BER}_{\max }$-reliable Gaussian sources as $\mathcal{R}=\left\{i \mid n_{i}>0\right\}$, the set of the indexes of the $\mathrm{BER}_{\max }$-unreliable Gaussian sources as $\mathcal{U}=\left\{i \mid n_{i}=0\right\}$, and their corresponding models as $m_{\mathcal{R}}=\left\{m_{i} \mid i \in \mathcal{R}\right\}=$ $\left\{m_{\mathcal{R}}^{1}, \ldots, m_{\mathcal{R}}^{|\mathcal{R}|}\right\}$ and $m_{\mathcal{U}}=\left\{m_{i} \mid i \in \mathcal{U}\right\}=\left\{m_{\mathcal{U}}^{1}, \ldots, m_{\mathcal{U}}^{|\mathcal{U}|}\right\}$ respectively, where $m$ is defined as in (20), and $|\mathcal{U}|+|\mathcal{R}|=$ $F$. Then, we can perform the procedure in Sect. IV-B3 in order to find the maximum number of reliable bits that we can encode with the Gaussian sources in $\mathcal{U}$, which we denote as $n_{\mathcal{U}}\left(m_{\mathcal{U}}, \mathrm{BER}_{\max }\right)$. The number of bits encoded by the Gaussian sources in $\mathcal{R}$ can be computed using (36) as $n_{\mathcal{R}}\left(m_{\mathcal{R}}, \mathrm{BER}_{\max }\right)=\sum_{i \in \mathcal{R}} n_{i}\left(m_{i}, \mathrm{BER}_{\max }\right)$. Therefore, we can compute the number of bits $n$ as:

$$
n_{\max }\left(m, \mathrm{BER}_{\max }\right)=n_{\mathcal{R}}\left(m_{\mathcal{R}}, \mathrm{BER}_{\max }\right)+n_{\mathcal{U}}\left(m_{\mathcal{U}}, \mathrm{BER}_{\max }\right) .
$$

We define the input $\boldsymbol{c}$ as the concatenation of the bits associated to the $\mathrm{BER}_{\max }$-reliable Gaussian sources $\boldsymbol{c}_{\mathcal{R}}$ and the bits associated to the $\mathrm{BER}_{\max }$-unreliable Gaussian sources $\boldsymbol{c}_{\mathcal{U}}$, i.e. $\boldsymbol{c}=\boldsymbol{c}_{\mathcal{R}} \mid \boldsymbol{c}_{\mathcal{U}}$, and we denote $\mathrm{ME}_{\mathcal{R}}, \mathrm{PM}_{\mathcal{R}}$ and $\mathrm{ME}_{\mathcal{U}}, \mathrm{PM}_{\mathcal{U}}$ as the metadata extractor and parametrized mapping functions of the model-based uncoupling construction described in Sect. IV-A and IV-B3 respectively, then we can define the corresponding metadata extraction function and parametrized mapping functions as follows:

$$
\begin{aligned}
\operatorname{ME}(m, \boldsymbol{c})= & p_{m, \boldsymbol{c}}=p_{m_{\mathcal{R}}^{1}, \boldsymbol{c}_{\mathcal{R}}^{1}}\|\cdots\| p_{m_{\mathcal{R}}^{n_{\mathcal{R}}}, \boldsymbol{c}_{\mathcal{R}}^{|\mathcal{R}|} \|} \| p_{m_{\mathcal{U}}, \boldsymbol{c}_{\mathcal{U}}} \\
= & \operatorname{ME}_{\mathcal{R}}\left(m_{\mathcal{R}}^{1}, \boldsymbol{c}_{\mathcal{R}}^{1}\right) \| \ldots \\
& \operatorname{ME}_{\mathcal{R}}\left(m_{\mathcal{R}}^{|\mathcal{R}|}, \boldsymbol{c}_{\mathcal{R}}^{|\mathcal{R}|}\right) \| \mathrm{ME}_{\mathcal{U}}\left(m_{\mathcal{U}}, \boldsymbol{c}_{\mathcal{U}}\right), \\
\operatorname{PM}\left(\boldsymbol{q}, p_{m, \boldsymbol{c}}\right)= & \operatorname{PM}_{\mathcal{R}}\left(q_{\mathcal{R}}^{1}, \boldsymbol{c}_{\mathcal{R}}^{1}\right) \| \ldots \\
& \operatorname{PM}_{\mathcal{R}}\left(q_{\mathcal{R}}^{|\mathcal{R}|}, \boldsymbol{c}_{\mathcal{R}}^{|\mathcal{R}|}\right) \| \operatorname{PM}_{\mathcal{U}}\left(\boldsymbol{q}_{\mathcal{U}}, \boldsymbol{c}_{\mathcal{U}}\right)
\end{aligned}
$$

where $\boldsymbol{c}=\left[\boldsymbol{c}_{\mathcal{R}}^{1}, \ldots, \boldsymbol{c}_{\mathcal{R}}^{|\mathcal{R}|}, \boldsymbol{c}_{\mathcal{U}}\right], \boldsymbol{q}=\left[q_{\mathcal{R}}^{1}, \ldots, q_{\mathcal{R}}^{|\mathcal{R}|}, \boldsymbol{q}_{\mathcal{U}}^{t}\right]^{t}$, and $q_{\mathcal{R}}^{i}$ and $m_{\mathcal{R}}^{i}$ are the verification sample and templates corresponding with the $i^{\text {th }}$ index in $\mathcal{R}$ respectively.

\section{General PROCEDURE TO OBTAIN OPTIMAL FUZZY EXTRACTORS FROM GAUSSIAN SOURCES}

In this section we explain how to design fuzzy extractors from Gaussian sources using operational requirements based on security and usability.

We assume that we can use the procedure from Sect. IV-C to get $n=n_{\max }\left(m, \mathrm{BER}_{\max }\right) \mathrm{BER}_{\max }$-reliable binary features. The operational requirements of a FE are as follows:

- The maximum allowed False Rejection Rate $\left(\mathrm{FRR}_{\max }\right)$. Taking into account the definition of the $(C, n, k, t)$-error correcting code, the False Rejection Rate, the $B R_{\max }$ and the code parameters are related as follows:

$$
\begin{aligned}
\operatorname{FRR}(n, t) & \leq \operatorname{Pr}\left\{X \sim \operatorname{Bi}\left(\operatorname{BER}_{\max }, n\right)>t\right\} \\
& =\sum_{i=t+1}^{n}\left(\begin{array}{l}
n \\
i
\end{array}\right) \operatorname{BER}_{\max }{ }^{i}\left(1-\mathrm{BER}_{\max }\right)^{n-i} .
\end{aligned}
$$

- The maximum allowed False Acceptance Rate (FAR $\left.\mathrm{Fax}_{\max }\right)$. Under the statistical assumptions we have considered for the Gaussian sources, this parameter is related with the error correcting code parameters. Using the definition of the code $(C, n, k, t)$ the False Acceptance Rate (FAR) for a honest adversary, who just samples a different Gaussian source and tries to impersonate the original one can be computed as follows:

$$
\operatorname{FAR}_{\text {hash }}(k)=\operatorname{Pr}\{X \sim \operatorname{Bi}(0.5, k)=0\}=2^{-k},
$$

whereas the FAR for a malicious adversary attacking the hash function in the Fuzzy Commitment scheme, it is computed as follows:

$$
\begin{aligned}
\operatorname{FAR}_{\text {honest }}(n, t) & =\operatorname{Pr}\{X \sim \operatorname{Bi}(0.5, n) \leq t\}=\frac{\sum_{i=0}^{t}\left(\begin{array}{c}
n \\
i
\end{array}\right)}{2^{n}} \\
& =\operatorname{FAR}_{\text {hash }}(k) \frac{\sum_{i=0}^{t}\left(\begin{array}{c}
n \\
i
\end{array}\right)}{2^{n-k}} .
\end{aligned}
$$

For any binary error correcting code, the number of error patterns that can be corrected is equal to the number of non-null syndromes, and therefore we have:

$$
\sum_{i=0}^{t}\left(\begin{array}{l}
n \\
i
\end{array}\right) \leq 2^{n-k} \Longrightarrow \operatorname{FAR}_{\text {hash }}(k) \geq \operatorname{FAR}_{\text {honest }}(n, t) \text {. }
$$

Attacks on the hash are statistically more likely to succeed than the honest attack on the fuzzy commitment:

$$
\begin{aligned}
\operatorname{FAR}(n, k, t) & =\max \left\{\operatorname{FAR}_{\text {hash }}(k), \operatorname{FAR}_{\text {honest }}(n, t)\right\} \\
& =\operatorname{FAR}_{\text {hash }}(k) .
\end{aligned}
$$

It is important to note that this definition of the FAR copes with both the Fuzzy-factor (represented by the honest adversary) and cryptographic (represented by the malicious adversary) securities.

We propose to adopt one of the following approaches, depending on the specific use case: 
- A security-driven approach, i.e. setting the parameter $F_{A R} R_{\max }$ as the security requirement, and then minimizing the FRR while ensuring FAR $\leq \mathrm{FAR}_{\max }$.

- A usability-driven approach, i.e. setting the parameter $\mathrm{FRR}_{\max }$ as the usability requirement, and then minimizing the FAR while ensuring $F R R \leq F R R_{\max }$.

\section{A. Security-driven approach}

This approach starts from setting the parameter FAR $\mathrm{max}_{\max }$ and then maximizes the FRR while ensuring FAR $\leq F A R_{\max }$.

For a given family of error correcting codes $\mathcal{E}$, the set of valid code parameters of $\mathcal{E}$ can be defined as:

$$
\mathcal{V}_{\mathcal{E}}=\{(n, k, t) \mid \exists(C, n, k, t) \in \mathcal{E}\} .
$$

Let us define the subset of $\mathrm{FAR}_{\max }$-secure code parameters:

$$
\mathcal{S}_{\mathcal{E}, \mathrm{FAR}_{\max }}=\left\{(n, k, t) \in \mathcal{V}_{\mathcal{E}} \mid \operatorname{FAR}(n, k, t) \leq \operatorname{FAR}_{\max }\right\} .
$$

We denote $n_{\max }\left(m, \mathrm{BER}_{\max }\right)$ as the maximum number of $\mathrm{BER}_{\max }$-reliable bits that we can extract from Gaussian sources with template $m$, as defined in (37). The procedure to compute this was shown in Sect. IV-C. Let us define the minimum possible BER for a given $n$ as:

$$
\operatorname{BER}_{\min }(m, n)=\min \left\{\operatorname{BER}_{\max } \mid n_{\max }\left(m, \mathrm{BER}_{\max }\right) \geq n\right\} .
$$

Denote the minimum achievable FRR for a given number $n$ of extracted bits and an error correction capability $t$ as:

$$
\operatorname{FRR}_{\min }(m, n, t)=\sum_{i=t+1}^{n}\left(\begin{array}{l}
n \\
i
\end{array}\right) \operatorname{BER}_{\min }(m, n)^{i}\left(1-\mathrm{BER}_{\min }(m, n)\right)^{n-i} .
$$

The optimal parameters of the code using the security-driven approach for a given security parameter $\mathrm{FAR}_{\max }$ can be defined as those providing the best possible usability (i.e. the minimum possible FRR) while abiding the security requirement (i.e., $\left.\operatorname{FAR}(n, k, t) \leq \operatorname{FAR}_{\max }\right)$ :

$$
(n, k, t)_{\mathrm{FAR}_{\text {max }} \text {-secure }}=\underset{(n, k, t) \in \mathcal{S}_{\mathcal{E}, \mathrm{FAR} \max }}{\operatorname{argmin}}\left\{\mathrm{FRR}_{\text {min }}(m, n, t)\right\} .
$$

\section{B. Usability-driven approach}

This approach starts from setting the parameter $F_{R R}$ max and then maximizes the FAR while ensuring $F R R \leq F R R_{\max }$. Therefore, we have to explore the solutions providing FRR $\leq$ $\mathrm{FRR}_{\max }$ and find the one providing the minimum FAR.

Using the definitions in (45), (47), and (48), we can define

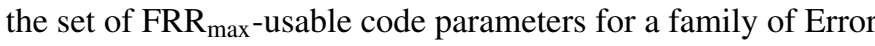
Correcting Codes $\mathcal{E}$ and Gaussian sources with template $m$ :

$$
\mathcal{U}_{\mathcal{F}, \mathcal{E}, \mathrm{FRR}_{\max }}=\left\{(n, k, t) \in \mathcal{V}_{\mathcal{E}} \mid \operatorname{FRR}_{\min }(m, n, t) \leq \operatorname{FRR}_{\max }\right\} .
$$

The optimal parameters of the code using the usability-driven approach for a given usability requirement $\mathrm{FRR}_{\max }$ is the one providing the best possible security (i.e. the lowest possible FAR) while abiding the usability parameter (i.e. FRR $\leq$ $\mathrm{FRR}_{\max }$ ), which can be defined as follows:

$$
(n, k, t)_{\mathrm{FRR}_{\text {max }} \text {-usable }}=\underset{(n, k, t) \in \mathcal{U}_{\mathcal{F}, \mathcal{E}, \mathrm{FRR} \max }}{\operatorname{argmin}}\{\mathrm{FAR}(n, k, t)\} .
$$

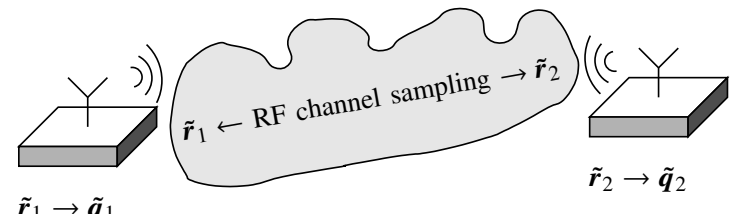

(1)
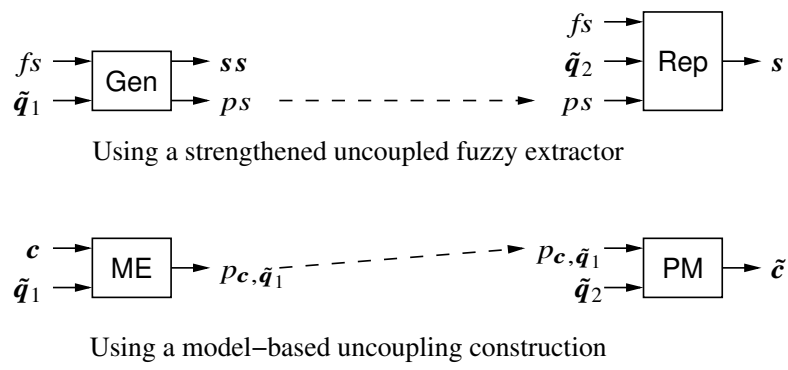

Fig. 6. Fuzzy extraction from RF channels using (1) the strengthened uncoupled fuzzy extractor, and (2) the model-based uncoupling construction.

\section{APPLICATION EXAMPLE: FUZZY EXTRACTION FROM RF CHANNELS}

In this section, we will demonstrate the usefulness of the model-based uncoupling construction and the strengthened uncoupled fuzzy extractor in illustrative use cases. Let us consider two parties communicating to each other through a RF channel. These parties observe the same channel in both directions, and this can be used to establish a key shared by both parties. The involved parties will sample the RF frequency response during the first phase of their communication, obtaining an estimate of the RF channel frequency response at multiple frequencies. Since these values are highly correlated for frequencies close to each other, first we decorrelate these by using Whitened Principal Component Analysis (WPCA).

We illustrate two different approaches (equivalent regarding FAR and FRR) to perform this key establishment:

a) Using the strengthened uncoupled fuzzy extractor: In this case, both parties get a common secret key in a setup phase, that can be used as a fixed authentication factor. The key establishment using a strengthened uncoupled fuzzy extractor is illustrated in Fig. 6(1). Both parties obtain their RF channel frequency estimates, and their corresponding channel representations. Then, one party runs the Gen procedure of the strengthened uncoupled fuzzy extractor shown in Fig. 1 using the secret key and its channel representation, stores the generated private string, and transmits the generated public string to the other party. The other party runs the Rep procedure using as inputs the received public string and its own channel representation, recovering the same private string. This private string can be used as a session key for communications among the parties. If the common secret key and the transmitted metadata is disclosed, there is no information on the decrypted metadata about the agreed session key, thus providing forward secrecy.

b) Using the model-based uncoupling construction: Linkability among different services is impossible in this use case, since the noisy factor will be different each time the system is used. Thus, it makes sense to avoid using 
the second authentication factor by relying on the modelbased uncoupling construction. In this case, illustrated in Fig. 6(2), there is no setup phase. During the key agreement, both parties obtain their RF channel frequency estimates, and their corresponding WPCA representations. Then, one party runs the ME procedure of the model-based uncoupling construction using its channel representation and a randomly chosen codeword from an error correcting code as inputs. The output metadata is transmitted to the other party. The other party uses the metadata and its own channel representation as inputs to the PM procedure of the model-based uncoupling construction, obtaining an estimate of the original codeword as output. The errors in this estimate are then corrected, and both parties derive a session key from it. Since the transmitted metadata is unrelated to the chosen codeword, the derived key can only be known by both parties.

A detailed security analysis of the proposed practical schemes is out of the scope of this paper, and their main purpose is to illustrate the advantages of using the proposed model-based uncoupling construction in terms of key length and FRR. However, it is worth to mention that the first approach provides the additional advantage of unlinkability, which is important when the continuous noisy source is persistent, e.g., a biometric or a PUF. The second approach is more practical for noisy sources derived from variant continuous noisy sources, e.g., derived from sensory data.

\section{A. Experimental Setup}

The radio channel measurements have been conducted using a 4-port vector network analyzer (VNA) to include multiple antenna orientations and polarizations. The measurement was conducted in an indoor environment in which the channel was characterized for the $2.4 \mathrm{GHz}$ ISM band, as this band is widely used for indoor applications. The measurement was carried out inside a meeting room of 7 by 12 meters, inside an office building. The meeting room was cleared of any furniture. The VNA used is a Keysight PNA-X N5242A 4-port network analyzer. The channel has been measured from 2.2 - $2.6095 \mathrm{GHz}$ at 4096 discrete frequency points, which corresponds to a frequency step-size of $100 \mathrm{kHz}$. Here we only use measurements relating to the $80 \mathrm{MHz}$ available in the $2.4 \mathrm{GHz}$ ISM-band, where we assume a stepsize of $500 \mathrm{kHz}$. Hence, 160 tones are used for feature extraction.

Two antenna pairs are used in the measurement, each pair having a horizontal and vertical polarized antenna. The antennas are quarter-wave whip antennas. The antennas are connected to the VNA using phase-stable and armored cables. For channel measurements, the cables and connectors are calibrated out using an Agilent E-cal, i.e. the measurement plain is at the antenna connector.

One of the antenna pairs has been placed at a fixed location in corner of the meeting room, while the other antenna pair was moved along a fine spatial grid through the meeting room resulting to 4 times 550 unique radio channel responses. The distance between antenna pairs varies between 1.5 and 10.6 meters.

Each transceiver obtains a channel characterization for each antenna pair, consisting of 160 amplitudes. We do not use the phase information in our experiment. Since each transceiver has 2 antennas, there are 4 different channels that we characterize, thus obtaining a multiple channel characterization $\mathbf{r}=$ $\left[r^{1}, \ldots, r^{L}\right]^{t}$ of lenght $L=4 \times 160=640$.

\section{B. Feature extraction: WPCA}

From a pool of $M$ RF channels frequency responses $R=$ $\left[\mathbf{r}_{1}|\cdots| \mathbf{r}_{M}\right]$, representing the population of possible frequency responses in the considered application scenarios, we calculate the empirical mean $\mu_{\mathbf{r}}=\frac{1}{M} \sum_{m=1}^{M} \mathbf{r}_{m}$ and the empirical covariance matrix $\Sigma=\frac{1}{M-1}\left(R-\boldsymbol{\mu}_{\mathbf{r}} \mathbf{1}_{M}\right)\left(R-\boldsymbol{\mu}_{\mathbf{r}} \mathbf{1}_{M}\right)^{t}$, where $\mathbf{1}_{M}$ is the column vector with $M$ ones. Let us denote by $\Gamma, \Lambda=\left[\mathbf{v}_{1}|\cdots| \mathbf{v}_{\Gamma}\right]$, and $\lambda=\left[\lambda_{1}, \ldots, \lambda_{\Gamma}\right]^{t}$ the rank of $\Sigma$, its eigenvectors matrix and its eigenvalues vector respectively. As a covariance matrix, $\Sigma$ is positive semi-definite, and thus $\lambda_{i}>0 \forall i \in\{1, \ldots, \Gamma\}$. Then, we define the Whitened Principal Analysis projection matrix as:

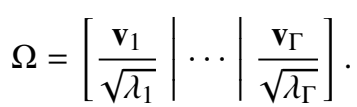

WPCA representations of a channel $\mathbf{r}$ can be computed as $\mathbf{q}=\Omega^{t}\left(\mathbf{r}-\mu_{\mathbf{r}}\right)$. It can be shown that population-wise, the WPCA channel representations $q_{i} \sim \mathcal{N}(0,1)$. However, since the characterizations $q_{i}$ corresponds to a sample from a random variable whose mean's pdf should be a standard Gaussian in the proposed model-based uncoupling construction for Gaussian features, we need to normalize each of the WPCA features. The frequency response estimates at each side are noisy, i.e. $\hat{r}_{i \text {,side* }}=r_{i}+\eta_{i \text {, side* }}$, where $r_{i}$ is the actual channel response value, and $\eta_{i \text {,side* }} \sim \mathcal{N}\left(0, \sigma^{2}\right)$ is a Gaussian additive noise. It can be shown that $q_{i} \sim \mathcal{N}\left(\mathbf{v}_{i}^{t}\left(\mathbf{r}-\mu_{\mathbf{r}}\right), \frac{\sigma^{2}}{\lambda_{i}}\right)$ for this specific channel. The normalized features are computed as $q_{i}^{\prime}=q_{i} / \sqrt{\sigma_{q_{i}}^{2}-\sigma^{2} / \lambda_{i}}$. After this, the population-wise pdf of the mean of $q_{i}^{\prime}$ is the standard Gaussian, and the pdf of the channel-specific $q_{i}^{\prime}$ also changes accordingly.

\section{Experimental Results}

In the experiments, we compared the proposed approaches using the model-based uncoupling construction for Gaussian features described in Section V with the baseline construction, which does not use any quantization helper data, as presented in Section IV-B. We use a simplified version of the usabilitydriven approach described in Section V-B, where we only use reliable Gaussian sources. This is reasonable in our use case, since we want the security level to be the same for all the RF channels. If we incorporate the unreliable Gaussian sources, as the number of bits extracted from them depends on the concrete value of the samples, this would no longer hold.

The codes are selected from the $\mathrm{BCH}$ family with length 127. We set the target usability in our experiment to $\mathrm{FRR}_{\max }=1 \%$. We added white Gaussian noise to the RF channels frequency responses in order to extend the comparison in performance between the proposed approaches and the baseline to different levels of Signal to Noise Ratio (SNR), which is the main factor affecting the performance of the fuzzy extraction. In both approaches we used BCH codes 


\begin{tabular}{cccccc} 
Approach & SNR[dB] & $n$ & $k$ & $t$ & FRR\% \\
\hline \hline Baseline & 22.05 & 127 & 50 & 13 & 4.41 \\
Proposed & (no added noise) & 127 & 120 & 1 & 6.82 \\
\hline Baseline & 21.71 & 127 & 50 & 13 & 4.45 \\
Proposed & & 127 & 120 & 1 & 6.77 \\
\hline Baseline & 19.47 & 127 & 43 & 14 & 4.00 \\
Proposed & & 127 & 113 & 2 & 5.73 \\
\hline Baseline & 17.23 & 127 & 36 & 15 & 3.73 \\
Proposed & & 127 & 106 & 3 & 5.41 \\
\hline Baseline & 15.79 & 127 & 29 & 21 & 4.09 \\
Proposed & & 127 & 92 & 5 & 4.68 \\
\hline Baseline & 14.22 & 127 & 29 & 21 & 3.55 \\
Proposed & & 127 & 78 & 7 & 3.64 \\
\hline Baseline & 12.47 & 127 & 29 & 21 & 3.00 \\
Proposed & & 127 & 64 & 10 & 3.00 \\
\hline Baseline & 10.66 & 127 & 22 & 23 & 2.41 \\
Proposed & & 127 & 43 & 14 & 2.41 \\
\hline Baseline & 8.79 & 127 & 15 & 27 & 1.14 \\
Proposed & & 127 & 29 & 21 & 1.18 \\
\hline Baseline & 6.88 & 127 & 8 & 31 & 1.00 \\
Proposed & & 127 & 15 & 27 & 1.05 \\
& & TABLE I & &
\end{tabular}

CODE PARAMETERS $(n, k, t)$, SNR, AND OBTAINED FRR FOR BOTH THE BASELINE AND PROPOSED CONSTRUCTIONS.

for error correction. We show the obtained code parameters and FRR for both the baseline and proposed approaches in Table I. One can see that using the proposed method results in a much longer security parameter $k$ for comparable FRR. Notice that the resulting FRR are in both cases higher than the target usability parameter $\mathrm{FRR}_{\max }$. This seems to be provoked by mismatch between the statistical assumptions on the noise nature. Although in most cases this noise behaves as i.i.d. Gaussian, matching with our assumptions, there are also outliers where the noise is of higher amplitude, and correlated among the different frequencies. However, the obtained FRR remains in an acceptable range for our proposed use case, since in the case that a false rejection is produced, the cost of repeating these procedures would be affordable. We can observe that when the added noise becomes more prevalent (for low values of SNR) the statistical assumptions on the noise become more accurate, and the obtained FRR gradually approaches the target FRR max $_{\text {. }}$.

\section{CONCLUSIONS}

In this paper, we formally introduced two new constructions, the model-based uncoupling construction dealing directly with noisy sources and producing metadata which (i) is unrelated to the discrete representation, (ii) guarantees the diversity of the discrete representation, and (iii) is useful for the conversion between the continuous and discrete domains; and the strengthened uncoupled fuzzy extractor, which specifies how the helper data derived by the model-based uncoupling construction and the additional fixed authentication factor must be handled for avoiding disclosure of information. The key extracted using the strengthened uncoupled fuzzy extractor is uncoupled to the noisy source, and can be used with cryptographic purposes. Moreover, the helper data do not leak any information about the key and is unlinkable after the encryption, which makes the strengthened uncoupled fuzzy extractor suitable for privacy-preserving applications.

These characteristics make the model-based uncoupling construction best suited for being used in cases where privacy of the noisy source characteristics or linkability of the noisy source among services may be a concern. On the other hand, the strengthened uncoupled fuzzy extractor is best suited for privacy preserving long-term cryptographic key derivation.

Furthermore, we presented the optimal model-based uncoupling construction for Gaussian sources, covering the full range of possible reliabilities. Based on these procedures, a unified design of key binding schemes from continuous data has been derived, both from a usability and security viewpoint.

Finally, we also demonstrate the effectiveness of the proposed techniques in a fuzzy extraction from RF channels use case. The proposed model-based uncoupling construction for Gaussian noisy sources almost doubles the amount of entropy extracted from the RF channels when compared to the baseline method, while providing also a lower FRR. These experiments demonstrate the advantages in terms of robustness of the proposed construction, which is able to provide a much better balance in terms of security (increased length of the derived key) and convenience (lower false negatives rate) when compared to fuzzy extractors from uniformly quantized continuous sources.

\section{APPENDIX A}

\section{BACKGROUND DEFINITIONS}

Definition A.1 (Error Correcting Codes). An $(C, n, k, t)$-error correcting code $C$ consists of two functions:

- ECC-enc : $C^{k} \rightarrow C^{n}$, takes as input a message $\mathbf{m} \in C^{k}$, and returns a codeword $\mathbf{w}=$ ECC-enc $(\mathbf{m}) \in C^{n}$.

- ECC-dec : $C^{n} \rightarrow C^{k}$, takes as input $\mathbf{w}^{\prime} \in C^{n}$, and returns a message $\mathbf{m} \in C^{k}$, if $\mathrm{d}_{C}\left(\mathbf{w}, \mathbf{w}^{\prime}\right) \leq t$ for $\mathbf{w}=$ ECC-enc $(\mathbf{m})$, and $\mathrm{d}_{C}(\cdot, \cdot)$ the distance in $C^{n}$.

Definition A.2 (Symmetric encryption scheme). A $(\mathcal{K}, \mathcal{P}, \mathcal{E}, \Lambda)$-symmetric encryption scheme is defined by a 3tuple (KeyGen, ENC, DEC) of functions. KeyGen : $\Lambda \rightarrow \mathcal{K}$ generates the encryption and decryption key $K \in \mathcal{K}$ using a security parameter $\lambda \in \Lambda$ as input, i.e., $K=\operatorname{KeyGen}(\lambda)$. ENC : $\mathcal{K} \times \mathcal{P} \rightarrow \mathcal{E}$ takes as input a plaintext $p \in \mathcal{P}$ and a key $K \in \mathcal{K}$ and outputs a ciphertext $e \in \mathcal{E}$, i.e., $e=\operatorname{ENC}_{K}(p)$. DEC : $\mathcal{K} \times \mathcal{E} \rightarrow \mathcal{P}$ takes as input a ciphertext $e \in \mathcal{E}$ and a key $K \in \mathcal{K}$ and outputs a plaintext $p \in \mathcal{P}$, i.e., $p=\operatorname{DEC}_{K}(e)$. If the encryption scheme is perfectly secure, then we drop $\Lambda$ from the notation.

Definition A.3 (Universal one-way hash functions (adapted from [28])). Let $\left\{n_{1}\right\}$ and $\left\{n_{0}\right\}$ be two increasing sequences such that $n_{0 i} \leq n_{1 i}, \forall i$, but $\exists q$ a polynomial, such that $q\left(n_{0 i}\right) \geq n_{1 i}$. Let $H_{k}$ be a collection of functions such that $\forall h \in H_{k}, h: C^{n_{1 k}} \rightarrow C^{n_{0 k}}$, and let $\mathcal{W}=\bigcup_{k} H_{k}$. Let $\mathcal{A}$ be a PPT adversary, that on input $k$ it outputs $x \in C^{n_{1 k}}$, an initial value. Then, given a random $h \in H_{k}, \mathcal{A}$ attempts to find $y \in C^{n_{1 k}}$ such that $h(y)=h(x)$, but $x \neq y$. $\mathcal{W}$ is called a family of universal one-way functions if for all $\mathcal{A}$ :

1) If $x \in C^{n_{1 k}}$ is $\mathcal{A}$ 's initial value, then $\operatorname{Pr}\{\mathcal{A}(h, x)=y, h(x)=h(y), x \neq y\}<\operatorname{negl}\left(n_{1 k}\right)$.

2) $\forall h \in H_{k} \exists$ a description of $h$ of length poly in $n_{1 k}$, s.t. given $h$ and $x, h(x)$ is computable in poly time.

3) $H_{k}$ is accessible: $\exists$ an algorithm $G$ s.t. on input $k, G$ generates unifomly at random a description of $h \in H_{k}$. 


\section{REFERENCES}

[1] Y. Dodis, R. Ostrovsky, L. Reyzin, and A. Smith, "Fuzzy extractors: How to generate strong keys from biometrics and other noisy data," SIAM J. Comput., vol. 38, no. 1, pp. 97-139, 2008.

[2] J. de Groot, B. Škorić, N. de Vreede, and J.-P. Linnartz, "Quantization in zero leakage helper data schemes," EURASIP J. on Advances in Signal Processing, vol. 2016:54, 2016

[3] K. Simoens, P. Tuyls, and B. Preneel, "Privacy weaknesses in biometric sketches," in 30th IEEE Symp. on Security and Privacy (S\&P 2009), 17-20 May 2009, Oakland, California, USA, 2009, pp. 188-203.

[4] "Information technology - Security techniques - Information security management systems - Requirements, ISO 27001:2013(E)," Int. Organization for Standardization, Geneva, CH, Standard, 2013.

[5] A. Juels and M. Wattenberg, "A fuzzy commitment scheme," in ACM CCS'99. ACM Press, 1999, pp. 28-36.

[6] V. V. T. Tong, H. Sibert, J. Lecoeur, and M. Girault, "Biometric fuzzy extractors made practical: A proposal based on fingercodes," in Proc. of the 2007 Int. Conf. on Advances in Biometrics, ser. ICB'07. SpringerVerlag, 2007, pp. 604-613.

[7] E. Argones Rua, E. Maiorana, J. Alba Castro, and P. Campisi, "Biometric template protection using universal background models: An application to online signature," IEEE Trans. Inf. Forensics Security, vol. 7, no. 1, pp. 269-282, Feb 2012.

[8] S. Billeb, C. Rathgeb, H. Reininger, K. Kasper, and C. Busch, "Biometric template protection for speaker recognition based on universal background models," IET Biometrics, vol. 4, no. 2, pp. 116-126, 2015.

[9] M. van der Veen, T. Kevenaar, G.-J. Schrijen, T. H. Akkermans, and F. Zuo, "Face biometrics with renewable templates," Proc. SPIE, vol. 6072, pp. 60720J-60720J-12, 2006.

[10] C. Rathgeb and A. Uhl, "Iris-biometric fuzzy commitment schemes under signal degradation," in Proc. of the 5th Int. Conf. on Image and Signal Processing, ser. ICISP'12. Springer-Verlag, 2012, pp. 217-225.

[11] E. Maiorana, D. Basi, and P. Campisi, "Biometric template protection using turbo codes and modulation constellations," in Workshop on Inform. Forensics and Security 2012, Tenerife, Spain, 2012.

[12] C. Bösch, J. Guajardo, A.-R. Sadeghi, J. Shokrollahi, and P. Tuyls, "Efficient helper data key extractor on FPGAs," in CHES 2008, ser. LNCS, E. Oswald and P. Rohatgi, Eds., vol. 5154. Springer Berlin Heidelberg, 2008, pp. 181-197.

[13] V. van der Leest, B. Preneel, and E. van der Sluis, Soft Decision Error Correction for Compact Memory-Based PUFs Using a Single Enrollment, ser. LNCS. Springer Berlin Heidelberg, 2012, vol. 7428, pp. 268-282.

[14] R. Maes, V. van der Leest, E. van der Sluis, and F. Willems, "Secure key generation from biased pufs: extended version," J. of Cryptographic Engineering, vol. 6, no. 2, pp. 121-137, Jun 2016.

[15] R. Maes, P. Tuyls, and I. Verbauwhede, Low-Overhead Implementation of a Soft Decision Helper Data Algorithm for SRAM PUFs, ser. LNCS. Springer Berlin Heidelberg, 2009, vol. 5747, pp. 332-347.

[16] R. Maes, P. Tulys, and I. Verbauwhede, "A soft decision helper data algorithm for SRAM PUFs," in 2009 IEEE Int. Symp. on Inform. Theory, 2009, pp. 2101-2105.

[17] M. D. Yu, R. Sowell, A. Singh, D. M'Raïhi, and S. Devadas, "Performance metrics and empirical results of a puf cryptographic key generation asic," in 2012 IEEE Int. Symp. on Hardware-Oriented Security and Trust, 2012, pp. 108-115.

[18] T. Ignatenko and F. M. J. Willems, "Information leakage in fuzzy commitment schemes," IEEE Trans. Inf. Forensics Security, vol. 5, no. 2, pp. 337-348, June 2010.

[19] T. Ignatenko, "Secret-key rates and privacy leakage in biometric systems," Ph.D. dissertation, Technische Universiteit Eindhoven, Eindhoven, 2009.

[20] E. A. Verbitskiy, P. Tuyls, C. Obi, B. Schoenmakers, and B. Skoric, "Key extraction from general nondiscrete signals," IEEE Trans. Inf. Forensics Security, vol. 5, no. 2, pp. 269-279, June 2010.

[21] M. Turk and A. Pentland, "Face recognition using eigenfaces," in Proc. of IEEE Conf. on Comput. Vision and Pattern Recognition, 1991, pp. 586-591.

[22] L. Huang, H. Zhuang, S. Morgera, and W. Zhang, "Multi-resolution pyramidal gabor-eigenface algorithm for face recognition," in Image and Graphics (ICIG'04), Third Int. Conf. on, December 2004, pp. 266-269.

[23] R. Kuhn, P. Nguyen, J.-C. Junqua, L. Goldwasser, N. Niedzielski, S. Fincke, K. Field, and M. Contolini, "Eigenvoices for Speaker Adaptation," in Int. Conf. on Spoken Language Processing, Dec. 1998.
[24] P. Kenny, G. Boulianne, P. Ouellet, and P. Dumouchel, "Joint factor analysis versus eigenchannels in speaker recognition," IEEE/ACM Trans. Audio, Speech, Language Process., vol. 15, no. 4, pp. 1435-1447, 2007.

[25] N. Dehak, P. J. Kenny, R. Dehak, P. Dumouchel, and P. Ouellet, "Frontend factor analysis for speaker verification," IEEE/ACM Trans. Audio, Speech, Language Process., vol. 19, no. 4, pp. 788-798, May 2011.

[26] R. E. Korf, "Multi-way number partitioning," in Proc. of the 21st Int. Jont Conf. on Artifical Intelligence, ser. IJCAI'09. Morgan Kaufmann Publishers Inc., 2009, pp. 538-543.

[27] R. E. Korf, E. L. Schreiber, and M. D. Moffitt, "Optimal sequential multi-way number partitioning." in Int. Symp. on Artificial Intelligence and Mathematics, 2014.

[28] M. Naor and M. Yung, "Universal one-way hash functions and their cryptographic applications," in Proc. of the Twenty-first Annual ACM Symp. on Theory of Computing, ser. STOC '89. ACM, 1989, pp. 3343 .

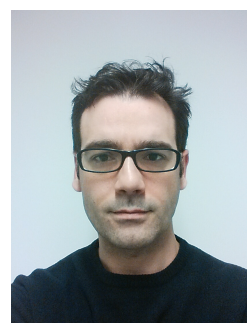

Enrique Argones Rúa received the M.Sc. and Ph.D. degrees in telecommunications engineering from the University of Vigo in Spain in 2003 and 2008 respectively. He was with Gradiant, ICT R\&D centre from 2012 to 2015 , where he was the technical leader of biometric technologies. Since 2015, he is with the imec-COSIC research group at the $\mathrm{KU}$ Leuven, Belgium.

His research interests are focused on the enhancement of privacy and security in authentication systems.

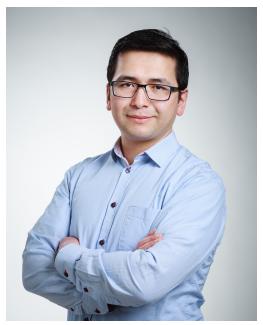

Aysajan Abidin received the B.Sc. degree in computational science from Xinjiang University in China in 2006, the M.Sc. degree in engineering mathematics from Chalmers University of Technology in Sweden in 2007, and the Ph.D. degree in information coding from Linköping University in Sweden in 2013. He worked on privacy-preserving biometric authentication as a post-doctoral researcher at Chalmers during 2014-2015.

Since 2015, he is a research fellow with the imecCOSIC research group, Department of Electrical Engineering (ESAT), KU Leuven. His research interests include information security, privacy, authentication, and applied cryptography.

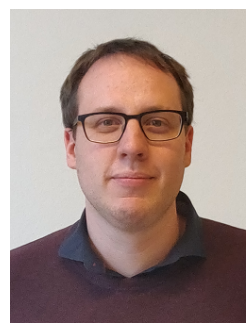

Roel Peeters was a postdoctoral researcher at the research group COSIC at KU Leuven (Belgium). He obtained a Master's degree in Electrical Engineering and a Phd in Engineering Sciences at KU Leuven in 2007 and 2012 respectively. His research interest are mainly in the analysis and design of cryptographic protocols. He authored more than 30 scientific publications and participated in various research projects. Currently he is the CTO of nextAuth, a company providing mobile user authentication solutions.

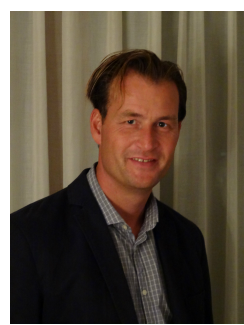

Jac Romme was born in Breda, the Netherlands, in 1975. He received the M.Sc. degree in 2000 in electrical engineering from Eindhoven University of Technology, The Netherlands, and a Ph.D. degree from Signal Processing and Speech Communication Laboratory (SPSC) at the Graz University of Technology, Austria. From 2000-2010, he was researcher for wireless radio systems at the IMST $\mathrm{GmbH}$, Germany. Since 2010 , he has been employed by Imec, the Netherlands. Currently, he is principle member of the technical staff of the IoT department. Since 2016 he is also a part-time Guest Researcher at Circuit and Systems Group at the TU Delft, the Netherlands. His research interests are radio communication and localization, (statistical) signal processing, Internet-ofThings and low-power IC design and modelling. 